ORP-56505

Revision NA

\title{
Memo, "Incorporation of HLW Glass Shell V2.0 into the Flowsheets," to ED Lee, CCN: 184905, October 20, 2009
}

Prepared for the U.S. Department of Energy

Assistant Secretary for Environmental Management

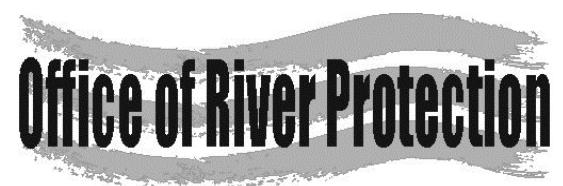

P.O. Box 450

Richland, Washington 99352 
ORP-56505

Revision NA

Memo, "Incorporation of HLW Glass Shell V2.0 into the
Flowsheets," to ED Lee, CCN: 184905, October 20, 2009

R. Gimpel

Bechtel National, Inc.

Date Published

October 2009

Prepared for the U.S. Department of Energy

Assistant Secretary for Environmental Management

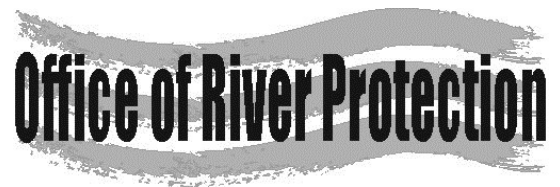

P.O. Box 450

Richland, Washington 99352

APPROVED

By Julia Raymer at 7:22 am, Dec 18, 2013

Release Approval

Date 
ORP-56505

Revision NA

TRADEMARK DISCLAIMER

Reference herein to any specific commercial product, process, or service by tradename, trademark, manufacturer, or otherwise, does not necessarily constitute or imply its endorsement, recommendation, or favoring by the United States Government or any agency thereof or its contractors or subcontractors.

This report has been reproduced from the best available copy.

Printed in the United States of America 


\section{Memorandum}

$\begin{array}{llc}\text { To: } & \text { Ernie Lee } & \text { Date: October 20, } 2009 \\ \text { From: } & \text { Rod Gimpel } & \text { CCN: } 184905 \\ \text { Ext: } & 371-3146 & \\ \text { Fax: } & 371-3204 & \\ \text { Subject: } & \text { INCORPORATION OF HLW GLASS SHELL V2.0 INTO THE FLOWSHEETS - } & \end{array}$

References: 1) CCN 153245, memorandum from Rod Gimpel to Ernie Lee, "New HLW Glass Chemistry Routine for Flowsheets - HLW Glass Shell v2.0." Dated June 30, 2009.

2) 24590-WTP-MCR-PET-09-0032, "New HLW Glass Chemistry Routine for Steady State Flowsheet - HLW Glass Shell v2.0"

3) 24590-WTP-MRQ-PET-09-0021, "New HLW Glass Chemistry Routine for Dynamic Flowsheet - HLW Glass Shell v2.0"

The new HLW Glass Shell v2.0 model properties (Reference 1) have been incorporated into the flowsheet models and validated. The HLW Glass Shell v2.0 logic presented in the attachment to Reference 1 was incorporated into the steady-state and dynamic flowsheet models per Reference 2 and Reference 3. The HLW Glass Shell v2.0 logic was also independently incorporated into an Excel spreadsheet, also referred to as the hand calculation. The results of the working steady-state and dynamic models were then compared against the spreadsheet. Differences in results between the flowsheet models and the spreadsheet indicated potential errors. Corrections were made in the flowsheet models and the spreadsheet until results of both were in close agreement. Changes and corrections were made to the attachment of Reference 1 during the validation. The changes and corrections are in noted the revised white paper, attached to this memorandum. Technical changes in that document are highlighted with revision bars in the margin.

In summary, the HLW Glass Shell v2.0 is a routine that estimates HLW glass volumes using the following glass property models:

- Nepheline,

- One-Percent Crystal Temperature $\left(\mathrm{T}_{1 \%}\right)$,

- Viscosity ( $\eta$ ),

- Product Consistency Tests (PCT) for boron, sodium, and lithium, and

- Liquidus Temperature, $\mathrm{T}_{\mathrm{L}}$. 
A database of the properties of waste glass and associated simulated waste glasses was collected and documented in PNNL 18501, Glass Property Data and Models for Estimating High-Level Waste Glass Volume (dated October 2009) and glass property models (cited above) were curve-fitted to the glass compositions. In addition to the use of the glass property models, glass composition constraints and rules, as recommend in PNNL 18501 and in other documents (as referenced in the attachment) were incorporated.

Any future revisions to the HLW Glass Chemistry Routine will be issued by Memorandum. Revisions will have "HLW Glass Shell" in the subject title so library database searches can be performed to find the latest version.

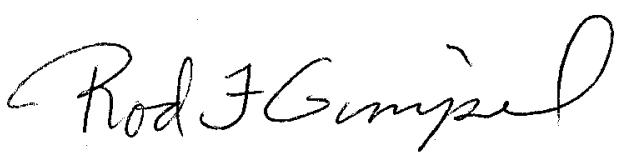

Rod Gimpel

Senior Process Engineer

Process Engineering and Technology

RFG/dlr

Attachment: HLW Glass Shell v2.0 as Incorporated into Flowsheets - October 2009

\section{Distribution}

Addressee

Mahoney, J. L.

Davis, S. A.

Deng, Y. N.

Jain, A.

Lowery, P. S.

Slaathaug, E. J.

Vienna, J. D.

Copy Coverage

PADC
MSIN

MS4-E2

MS4-E2

MS4-E2

MS4-E2

MS4-E2

MS4-E2

MS4-B2

MSIN

MS19-A 


\section{HLW Glass Shell v2.0 as Incorporated into Flowsheets October 2009}

Efforts are being made to increase the efficiency and decrease the cost of vitrifying radioactive waste stored in tanks at the U.S. Department of Energy Hanford Site. The compositions of acceptable and processable high-level waste (HLW) glasses need to be optimized to minimize the waste-form volume and, hence, to reduce cost. A database of glass properties of waste glass and associated simulated waste glasses was collected and documented in PNNL 18501, Glass Property Data and Models for Estimating High-Level Waste Glass Volume and glass property models were curve-fitted to the glass compositions. A routine was developed that estimates HLW glass volumes using the following glass property models:

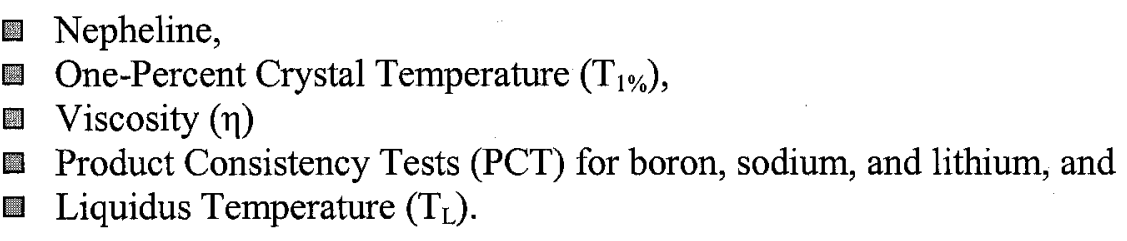

The routine, commonly called the $H L W$ Glass Shell, is presented in this document.

In addition to the use of the glass property models, glass composition constraints and rules, as recommend in PNNL 18501 and in other documents (as referenced in this report) were incorporated. This new version of the HLW Glass Shell should generally estimate higher waste loading in the HLW glass than previous versions.

\section{Methodology}

The objective for the HLW Glass Shell is to provide a reasonable and probable HLW glass composition using a single-pass calculation routine. The major steps in the routine are:

Estimate the mass of HLW glass by determining the most limiting constituent or combination of constituents in the waste.

(1) Probable glass formers are chosen to occupy the volume of glass that is not waste. The waste and chosen glass formers constitute the initial glass recipe known as the PreGlass.

The PreGlass composition is checked against the various glass property models.

a Changes are made to the PreGlass composition/glass formers as necessary to bring the glass composition into compliance with the various glass properties.

- Swapping glass formers is tried first to bring the glass composition into compliance because this goes not change the waste loading in the glass.

$\square$ If swapping does not work, the PreGlass is blended with another glass (called the Dilution Glass) until it becomes compliant. The Dilution Glass is low in the property that limits the PreGlass. The lowest property-limiting Dilution Glass that also meets the other properties, as well, is used to limit its impact to waste loading. 
The PreGlass is checked against the glass property models in a systematic order and changes made to the glass formers. Each succeeding change in the glass formers will change the PreGlass values for previously checked glass property models, but the changes should be in the positive direction for the previous glass property models. The order of checking by the glass property models is:

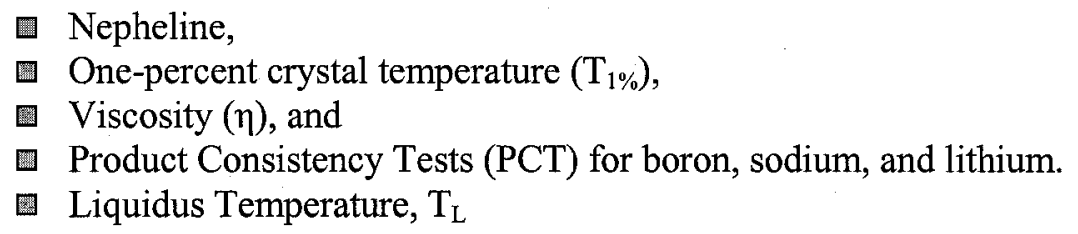

\section{Prime Variables - Oxides that make HLW glass}

Elements that make glass are most easily handled as oxides in glass formulations. Therefore all metal elements need to be converted to their oxide for calculation. Anions are also reduced to an oxide form. This allows for compounds to be handled as separate constituents and maintain mass balance. For example, calcium sulfate, calcium phosphate, and calcium nitrate would be handled as follows:

Equation 1

$\mathrm{CaSO}_{2} \rightarrow \mathrm{CaO}+\mathrm{SO}_{3}$

$\mathrm{Ca}_{3}\left(\mathrm{PO}_{4}\right)_{2} \rightarrow 3 \mathrm{CaO}+\mathrm{P}_{2} \mathrm{O}_{5}$

$\mathrm{Ca}\left(\mathrm{NO}_{3}\right)_{2} \rightarrow \mathrm{CaO}+\mathrm{N}_{2} \mathrm{O}_{5} \uparrow$ (or $\mathrm{NOx}$ gases $\uparrow$ )

Notice that nitrates (Equation 1) break down, due to the heat, and form $\mathrm{NO}_{\mathrm{x}}$ gases that leave the glass.

For convenience in calculations, the waste oxides that have a glass former counterpart are traced separately. Therefore, the amount of waste in the batch is represented in Equation 2 as follows.

Equation 2

$\mathrm{M}_{\mathrm{waste}}=\mathrm{M}_{\mathrm{A} 12 \mathrm{O} 3}+\mathrm{M}_{\mathrm{B} 2 \mathrm{O} 3}+\mathrm{M}_{\mathrm{Fe} 2 \mathrm{O} 3}+\mathrm{M}_{\mathrm{Li} 2 \mathrm{O}}+\mathrm{M}_{\mathrm{Na} 2 \mathrm{O}}{ }^{1}+\mathrm{M}_{\mathrm{SiO} 2}+\mathrm{M}_{\mathrm{others}}+\mathrm{M}_{\mathrm{rad}}$

where: $\mathrm{M}_{\mathrm{others}}=\sum \mathrm{M}_{i}$

$i=$ batch waste consituents as oxides for $\mathrm{Ag}_{2} \mathrm{O}, \mathrm{As}_{2} \mathrm{O}_{5}, \mathrm{BaO}, \mathrm{BeO}, \mathrm{Bi}_{2} \mathrm{O}_{3}, \mathrm{CaO}, \mathrm{CdO}, \mathrm{Ce}_{2} \mathrm{O}_{3}, \mathrm{Cl}$, $\mathrm{CoO}, \mathrm{Cr}_{2} \mathrm{O}_{3}, \mathrm{Cs}_{2} \mathrm{O}, \mathrm{CuO}, \mathrm{Dy}_{2} \mathrm{O}_{3}, \mathrm{Eu}_{2} \mathrm{O}_{3}, \mathrm{~F}, \mathrm{Gd}_{2} \mathrm{O}_{3}, \mathrm{HfO}_{2}, \mathrm{HgO},{ }^{z} \mathrm{~K}_{2} \mathrm{O}, \mathrm{La}_{2} \mathrm{O}_{3}, \mathrm{MgO}, \mathrm{MnO}^{3}, \mathrm{MoO}_{3}$, । $\mathrm{Nd}_{2} \mathrm{O}_{3}, \mathrm{NiO}, \mathrm{P}_{2} \mathrm{O}_{5}, \mathrm{PbO}, \mathrm{PdO}, \mathrm{Pr}_{2} \mathrm{O}_{3}, \mathrm{Rb}_{2} \mathrm{O}, \mathrm{Re}_{2} \mathrm{O}_{7}, \mathrm{Rh}_{2} \mathrm{O}_{3}, \mathrm{RuO}_{2}, \mathrm{Sb}_{2} \mathrm{O}_{3}, \mathrm{SeO}_{2}, \mathrm{SO}_{3}, \mathrm{Sm}_{2} \mathrm{O}_{3}$, $\mathrm{SnO}_{2}, \mathrm{SrO}, \mathrm{Tc}_{2} \mathrm{O}_{7}, \mathrm{TeO}_{2}, \mathrm{ThO}_{2}, \mathrm{TiO}_{2}, \mathrm{Tl}_{2} \mathrm{O}, \mathrm{UO}_{3}, \mathrm{~V}_{2} \mathrm{O}_{5}, \mathrm{WO}_{3}, \mathrm{Y}_{2} \mathrm{O}_{3}, \mathrm{ZnO}$, and $\mathrm{ZrO}_{2}$.

$\mathrm{M}_{\mathrm{rad}}=$ Mass of radionuclides tracked as oxides in strict accounting models to maintain material balance. However, this is not needed for estimating glass formulation and glass quantities, and can be considered zero for such.

The halides $(\mathrm{Cl}, \mathrm{F}$, and $\mathrm{I})$ are not tracked as oxides because they actually substitute oxide in the glass. For example, $\mathrm{SiO}_{2}$ becomes $\mathrm{SiO}_{3 / 2} \mathrm{Cl}, \mathrm{SiOCl}_{2}$, etc. This reaction needs to be accounted in strict accounting

\footnotetext{
${ }^{1}$ The dynamic flowsheet (G2) also tracks a second form of sodium called Process Sodium (Nap) that is added in the WTP process. $\mathrm{Nap}_{2} \mathrm{O}$ shall be included with waste $\mathrm{Na}_{2} \mathrm{O}$ when determining glass chemistry and volumes.

${ }^{2} \mathrm{HgO}$ is a waste metal oxide that is not included in list because glass has little or no affinity to mercury and essentially all mercury leaves the melter and enters the offgas.

${ }^{3}$ The dynamic flowsheet (G2) also tracks a second form of manganese called Process Manganese (Mnp) that is added in the WTP process. MnpO shall be included with waste $\mathrm{MnO}$ when determining glass chemistry and volumes.
} 
models like ACM. Bookkeeping is much easier if all the oxide is assumed to be replaced by the halide (i.e., $\mathrm{SiCl}_{4}$ ) even though these silicon halides are gases at room temperatures. This reaction actually happens in molten glass and some of halide leaves as silicon halide gas. This helps explain the semivolatile nature of halides in glass.

Table 1 shows the main variables that the HLW glass chemistry subroutines will solve for and define. The amount of glass formers is determined on the amounts and ratios of constituents of the waste oxides.

Table 1 Prime Variables

\begin{tabular}{|l|l|l|}
\hline Name (mass Fraction) & Name (mass) & Represents \\
\hline $\mathrm{W}_{\mathrm{L}}$ & $\mathrm{M}_{\mathrm{W}}$ & Waste loading or waste oxides \\
\hline 1 or $100 \mathrm{wt} \%$ & $\mathrm{M}_{\mathrm{Glass}}$ & Mass of Glass \\
\hline $\mathrm{A}_{\mathrm{A} 1203}$ & $\mathrm{GF}_{\mathrm{A} 1203}$ & Aluminum oxide additive (Alumina) \\
\hline $\mathrm{A}_{\mathrm{B} 203}$ & $\mathrm{GF}_{\mathrm{B} 203}$ & Boron oxide additive (Boria) \\
\hline $\mathrm{A}_{\mathrm{Fe} 203}$ & $\mathrm{GF}_{\mathrm{Fe} 203}$ & Iron oxide additive \\
\hline $\mathrm{A}_{\mathrm{L} 20}$ & $\mathrm{GF}_{\mathrm{Li2O}}$ & Lithium oxide additive (Lithia) \\
\hline $\mathrm{A}_{\mathrm{Na} 20}$ & $\mathrm{GF}_{\mathrm{Na} 2 \mathrm{O}}$ & Sodium oxide additive (Sodia) \\
\hline $\mathrm{A}_{\mathrm{SiO} 2}$ & $\mathrm{GF}_{\mathrm{SiO} 2}$ & Silicon oxide (Silica) \\
\hline
\end{tabular}

Note that tables below may use percentages because formulations and comparisons are visualized better this way. However, equivalent decimal fractions shall be used in the equations and computer programming unless otherwise noted.

Zinc oxide is added to LAW glass to protect the melter refractories from corrosion/erosion. Around 1 to $2 \mathrm{wt} \% \mathrm{ZnO}$ is used to form spinels that collect on the surface LAW melter wall refractories. The spinels then protect the refractories from corrosion. However, it is assumed that the HLW glasses, themselves, naturally produce enough spinels to protect HLW melter wall refractories. Therefore,

$\mathrm{A}_{\mathrm{ZnO}}=0.0 ; \quad \mathrm{GF}_{\mathrm{Zn}}=0.0$

$\mathrm{ZnO}$ will be treated as a waste oxide in the $\mathrm{HLW}$ glass chemistry subroutines.

\section{Prime Equation}

Equation 3

$1=W_{\mathrm{L}}+\mathrm{A}_{\mathrm{A} 12 \mathrm{O} 3}+\mathrm{A}_{\mathrm{B} 2 \mathrm{O} 3}+\mathrm{A}_{\mathrm{Fe} 2 \mathrm{O} 3}+\mathrm{A}_{\mathrm{L} \mathrm{i} 2 \mathrm{O}}+\mathrm{A}_{\mathrm{Na} 2 \mathrm{O}}+\mathrm{A}_{\mathrm{SiO} 2}$

\section{Objective}

Maximize $\mathrm{W}_{\mathrm{L}}$ while still meeting glass property model limits.

\section{Glass Amounts}

The amount of glass made from a batch is the summation of the waste oxides and glass formers added.

$$
\begin{aligned}
& \text { Equation } 4 \\
& \mathrm{M}_{\text {glass }}=\mathrm{M}_{\mathrm{A} 203}+\mathrm{M}_{\mathrm{B} 2 \mathrm{O} 3}+\mathrm{M}_{\mathrm{re} 2 \mathrm{O} 3}+\mathrm{M}_{\mathrm{Li2O}}+\mathrm{M}_{\mathrm{Na} 2 \mathrm{O}}+\mathrm{M}_{\mathrm{SiO} 2}+\mathrm{M}_{\mathrm{others}}+ \\
& \mathrm{GF}_{\mathrm{A} 203}+\mathrm{GF}_{\mathrm{B} 2 \mathrm{O} 3}+\mathrm{GF}_{\mathrm{Fe} 2 \mathrm{O} 3}+\mathrm{GF}_{\mathrm{Li2O}}+\mathrm{GF}_{\mathrm{Na} 2 \mathrm{O}}+\mathrm{GF}_{\mathrm{SiO} 2}
\end{aligned}
$$

The amount of glass can be determined by knowing a constituent oxide mass and its concentration in the glass as shown in Equation 5. 
Equation 5

$\mathrm{M}_{\mathrm{glass}}=\mathrm{M}_{i} / \mathrm{W}_{i}=\mathrm{GF}_{i} / \mathrm{A}_{i}=\left(\mathrm{M}_{i}+\mathrm{GF}_{i}\right) / \mathrm{g}_{i}$

where: $\mathrm{W}_{i}$ is the waste loading of a waste constituent in the glass as mass fraction, $\mathrm{kg} / \mathrm{kg}$. $\mathrm{g}_{\mathrm{i}}$ is the mass concentration of constituent in the glass as mass fraction, $\mathrm{kg} / \mathrm{kg}$.

\section{Bounding Conditions}

Only one constituent or combination of constituents should meet bounding conditions in the glass. As such, the glass is said to be constrained by that condition because the glass is known or considered to be incapable of tolerating any more of that constituent or combination. The glass should contain less than allowed for all the other constituents. The bounding conditions and their values are listed in Table 2 . The bounding constraints are used in determining a preliminary estimate of the amount of glass that will be made called the PreGlass. The PreGlass recipe is tested in various glass models to determine if it will make a good glass. If not, changes will be made to bring the glass in line with glass model constraints.

Table 2 HLW Glass Bounding Conditions, $\mathrm{BC}_{i}$

\begin{tabular}{|c|c|c|c|}
\hline Name & Constituent value or Series & Upper Limit $^{(a)}$, wt $\%$ & Rule \\
\hline $\mathrm{BC}_{1}$ & $\mathrm{M}_{\mathrm{A} 1203}$ & 20 & Aluminum Validity Region ${ }^{(c)}$ \\
\hline $\mathrm{BC}_{2}$ & $\mathrm{M}_{\mathrm{Bi203}}$ & 3.2 & Bismuth Validity Region $^{(c)}$ \\
\hline $\mathrm{BC}_{3}$ & $\mathrm{M}_{\mathrm{CaO}}$ & 7. & Calcium Validity Region ${ }^{(c)}$ \\
\hline $\mathrm{BC}_{4}$ & $\mathrm{M}_{\mathrm{CdO}}$ & 1.5 & Cadmium Validity Region ${ }^{(c)}$ \\
\hline $\mathrm{BC}_{5}$ & $\mathrm{M}_{\mathrm{Cl}}^{\left({ }^{(a)}\right.}$ & 0.5 & Chloride Constraint ${ }^{\text {(c) }}$ \\
\hline $\mathrm{BC}_{6}$ & $\overline{\mathrm{M}}_{\mathrm{Cr} 2 \mathrm{O} 3}$ & 1.2 & Chromium Validity Region ${ }^{(\mathrm{c})}$ \\
\hline $\mathrm{BC}_{7}$ & $\mathrm{M}_{\mathrm{F}}^{(\mathrm{a})}$ & 2. & Fluoride Constraint $^{(\mathrm{c})}$ \\
\hline $\mathrm{BC}_{8}$ & $\mathrm{M}_{\mathrm{Fe} 2 \mathrm{O} 3}$ & 17.4 & Iron Validity Region ${ }^{(\mathrm{c})}$ \\
\hline $\mathrm{BC}_{9}$ & $\mathrm{M}_{\mathrm{K} 2 \mathrm{O}}$ & 6. & Potassium Validity Region ${ }^{(c)}$ \\
\hline $\mathrm{BC}_{10}$ & $\mathrm{M}_{\mathrm{MgO}}$ & 6. & Magnesium Validity Region ${ }^{(\mathrm{c})}$ \\
\hline $\mathrm{BC}_{11}$ & $\mathrm{M}_{\mathrm{MnO}}$ & 7. & Manganese Validity Region ${ }^{(c)}$ \\
\hline $\mathrm{BC}_{12}$ & $\mathrm{M}_{\mathrm{Na} 2 \mathrm{O}}$ & 21.4 & Sodium Validity Region ${ }^{(c)}$ \\
\hline $\mathrm{BC}_{13}$ & $\mathrm{M}_{\mathrm{NiO}}$ & 3. & Nickel Validity Region ${ }^{(c)}$ \\
\hline $\mathrm{BC}_{14}$ & $\mathrm{M}_{\mathrm{NM}}=\mathrm{M}_{\mathrm{Rh} 203}+\mathrm{M}_{\mathrm{RuO} 2}+\mathrm{M}_{\mathrm{PdO}}$ & 0.25 & Noble Metal Constraint ${ }^{(\mathrm{c})}$ \\
\hline $\mathrm{BC}_{15}$ & $\mathrm{M}_{\mathrm{p2O}}$ & 2.5 & Phosphate Constraint ${ }^{(\mathrm{c})}$ \\
\hline $\mathrm{BC}_{16}$ & $\mathrm{M}_{\mathrm{P2O} 5} \times \mathrm{M}_{\mathrm{CaO}}$ & $6.5^{(\mathrm{c})}$ & Phosphate Constraint ${ }^{(c)}$ \\
\hline $\mathrm{BC}_{17}$ & $\mathrm{M}_{\mathrm{PbO}}$ & 5. & Trace Elements of Concern ${ }^{(c)}$ \\
\hline $\mathrm{BC}_{18}$ & $\mathrm{M}_{\mathrm{SiO} 2}$ & 53. & Silica Validity Region ${ }^{(\mathrm{c})}$ \\
\hline $\mathrm{BC}_{19}$ & $\mathrm{M}_{\mathrm{SO}^{(a)}}{ }^{(2)}$ & 0.5 & Sulfate Constraint ${ }^{(c)}$ \\
\hline $\mathrm{BC}_{20}$ & $\mathrm{M}_{\mathrm{SrO}}$ & 4.5 & High Liquidus Temperature $^{(d)}$ \\
\hline $\mathrm{BC}_{21}$ & $\mathrm{M}_{\mathrm{ThO} 2}$ & 6. & Thorium Validity Region ${ }^{(\mathrm{c})}$ \\
\hline $\mathrm{BC}_{22}$ & $\mathrm{M}_{\mathrm{UO3}}$ & 6.3 & Uranium Validity Region $^{(\mathrm{c})}$ \\
\hline $\mathrm{BC}_{23}$ & $\mathrm{M}_{\mathrm{Zr2O}}$ & 13.5 & Non-Spinel Rule $C^{(c)}$ \\
\hline $\mathrm{BC}_{24}$ & $\mathrm{M}_{\text {minors }}(\mathrm{b})$ & 4.5 & Minors Validity Region ${ }^{(c)}$ \\
\hline
\end{tabular}

(a) The melter feed concentration before applying melter decontamination factors (DF's) or glass retention factors. Also known as target concentration.

(b) $\mathrm{M}_{\mathrm{others}}-\mathrm{M}_{\mathrm{Bi2O3}}-\mathrm{M}_{\mathrm{CaO}}-\mathrm{M}_{\mathrm{CdO}}-\mathrm{M}_{\mathrm{C} 2 \mathrm{O} 3}-\mathrm{M}_{\mathrm{K} 2 \mathrm{O}}-\mathrm{M}_{\mathrm{MgO}}-\mathrm{M}_{\mathrm{MnO}}-\mathrm{M}_{\mathrm{NiO}}-\mathrm{M}_{\mathrm{PbO}}-\mathrm{M}_{\mathrm{SO} 3}-\mathrm{M}_{\mathrm{SrO}}-\mathrm{M}_{\mathrm{ThO} 2}-\mathrm{M}_{\mathrm{Ti} 2 \mathrm{O}}-$ $\mathrm{M}_{\mathrm{UO} 3}-\mathrm{M}_{\mathrm{ZrO} 2}$

(c) PNNL-18501, Glass Property Data and Models for Estimating High-Level Glass Volume, J.D. Vienna, et. al., October 2009.

(d) Even though PNNL-18501 validity range is $10.1 \mathrm{wt} \%$ the HLW glass shell can not handle this high value. SrO affects the Liquidus Temperature, $T_{L}$, several factors more than any other oxide and causes negative $\mathrm{B}_{2} \mathrm{O}_{3}$ values in attempts to adjust very high $\mathrm{T}_{\mathrm{L}}$ values due to high $\mathrm{SrO}$. Therefore, the $\mathrm{BC}$ value for $\mathrm{SrO}$ is limited as shown.

(e) The units of this values are not wt \% as are shown with the other values. Its units are wt $\%$ squared. 
Non-spinel rules as given in CCN 184900, Non-Spinel Phase Rule - Supersedes CCN 170601, John Vienna, September 16,2008, were not incorporated into the HLW Glass Shell because it was felt that the new Liquidus Temperature model covers the intent of the non-spinel rules dealing with combined glass concentrations of $\mathrm{Al}_{2} \mathrm{O}_{3}, \mathrm{ThO}_{2}$, and $\mathrm{ZrO}_{2}$.

\section{Preliminary Estimate of Glass Amount, PreGlass}

The preliminary estimate of the HLW glass, or PreGlass $\mathrm{M}_{\text {Glass.pl }}$, is determined by the most constrained constituent or bounding condition listed in Table 2. This is determined by comparing each of the waste constituents to their corresponding limits.

Equation 6

$\mathrm{BC}_{\mathrm{Max}} \quad=\mathrm{MAX}\left(\mathrm{M}_{1} / \mathrm{BC}_{1}, \ldots \mathrm{M}_{15} / \mathrm{BC}_{15},\left(\mathrm{M}_{16} / \mathrm{BC}_{16}\right)^{0.5}, \mathrm{M}_{17} / \mathrm{BC}_{17}, \ldots \mathrm{M}_{24} / \mathrm{BC}_{24}\right)$

Note: Percentages are used as decimal fractions. $\mathrm{BC}_{16}$ value to use in Equation 6 is 0.00065 because its units are mass fraction squared. $\mathrm{M}_{16}$ is $\left(\mathrm{M}_{\mathrm{P} 205} * \mathrm{M}_{\mathrm{CaO}}\right)$ as shown in Table 2. Also, logic for equations is written in Excel or Basic formats.

Equation 7

$\mathrm{BC}_{\mathrm{Flag}} \quad=\mathrm{BC}_{i}$ number or name of $\mathrm{BC}_{\mathrm{Max}}$ (i.e, if iron is limiting, $\mathrm{BC}_{\mathrm{Flag}}=8$ or Iron

(See Table 2)

GlassProp $_{0}=0$

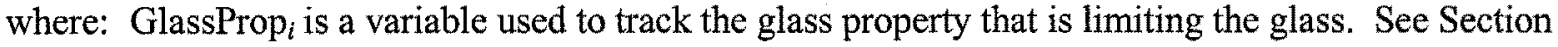
GlassProp Number for details. (Table 13)

$i$ is the $i^{\text {th }}$ variable in the GlassProp series. There are 12 variables in the series, i.e., $i=0 \ldots 11$.

\section{PreGlass Recipes}

The glass formers at this stage are only estimated quantities used for evaluation and correction in the property models. These estimated quantities will have a ".p" with a number placed behind the value name to signify they are pre-estimates. The number means that it is the $i$ th estimate for the value. For example, $\mathrm{GF}_{\mathrm{SiO2} \text {.p1 }}$ is the first estimated quantity for the glass former/additive silica. Glass properties are checked using this value and then changed if necessary. Estimated Glass mass can change as well and it follows the same labeling convention.

\section{PreGlass Glass Formers}

Since most of the batches coming from the tank farms contain excess amounts of aluminum and some amount of leaching will be required, it does not make sense to add aluminum glass former to the recipe unless a glass property model absolutely requires it or the PreGlass contains so little aluminum it is below the validity levels of the developed glass models, which is $1.9 \mathrm{wt} \%$. Other glass formers have low validity levels as well. Equation 8 through Equation 17 provides the minimum glass former amounts for those glass formers that have minimum constraints. The initial glass former coefficients for the PreGlass are given in Table 3. Equation 15 checks to see if the estimated amount of silica glass former calculated in Equation 14 results in the glass exceeding the maximum glass concentration $\left(\mathrm{BC}_{18}\right)$. If so, Equation 15 and Equation 16 swap Glass Former $\mathrm{Fe}_{2} \mathrm{O}_{3}$ for Glass Former $\mathrm{SiO}_{2}$. The mass of the PreGlass $\left(\mathrm{M}_{\mathrm{Glass} . \mathrm{pl}}\right)$ is the sum of the waste and glass formers, as calculated in Equation 17. 
Table 3 Coefficients for Initial Glass Formers (igf

\begin{tabular}{|c|c|c|c|}
\hline Name & Use with GF & Basis & Value \\
\hline igf $_{1}$ & $\mathrm{GF}_{\mathrm{Al203} . \mathrm{pl}}$ & Minimum & 0.04 \\
\hline igf $_{2}$ & $\mathrm{GF}_{\mathrm{B} 203 . \mathrm{pl}}$ & Initial value & 0.20 \\
\hline igf $_{3}$ & $\mathrm{GF}_{\mathrm{Fe} 203 . \mathrm{p0}}$ & Initial value & 0.03 \\
\hline igf $_{4}$ & $\mathrm{GF}_{\mathrm{Li} 20 . \mathrm{p} 1}$ & Initial value & 0.0133 \\
\hline igf $_{5}$ & $\mathrm{GF}_{\text {Na220.p1 }}$ & Initial value & 0.0925 \\
\hline igf $_{6 \mathrm{a}}$ & $\mathrm{GF}_{\text {Si02.p0 }}$ & Minimum $^{5}$ & 0.35 \\
\hline igf $_{6 \mathrm{~b}}$ & $\mathrm{GF}_{\text {Si02.p0 }}$ & $\mathrm{Al}, \mathrm{Cr}, \mathrm{PO}_{4}$ use $^{5}$ & 0.41 \\
\hline
\end{tabular}

Equation 8

$\mathrm{GF}_{\mathrm{Al} 2 \mathrm{O} 3 \mathrm{pl} 1}=\mathrm{IF}\left(\mathrm{M}_{\mathrm{Al2O} 3} / \mathrm{BC}_{\mathrm{Max}}<\operatorname{igf}_{1}, \operatorname{igf}_{1} * \mathrm{BC}_{\mathrm{Max}}-\mathrm{M}_{\mathrm{Al} 2 \mathrm{O} 3}, 0.0\right)$

Fouation 9

$\mathrm{GF}_{\mathrm{B} 203 . \mathrm{pl}}$

$$
=\operatorname{MAX}\left(\operatorname{igf}_{2} * \mathrm{BC}_{\mathrm{Max}}-\mathrm{M}_{\mathrm{B} 203}, 0\right)
$$

Fquation 10

$\mathrm{GF}_{\mathrm{Fe203.p0}}$

$$
=\operatorname{MAX}\left(\operatorname{igf}_{3} * \mathrm{BC}_{\mathrm{Max}}-\mathrm{M}_{\mathrm{Fe} 203}, 0\right)
$$

Equation 11

$\mathrm{GF}_{\mathrm{Li} 2 \mathrm{O}, p 1}$

$$
=\operatorname{MAX}\left(\operatorname{igf}_{4} * \mathrm{BC}_{\mathrm{Max}}-\mathrm{M}_{\mathrm{Li2O}}, 0\right)
$$

Equation 12

$\mathrm{GF}_{\mathrm{Na} 20 . \mathrm{p} 1}$

$$
=\operatorname{MAX}\left(\operatorname{igf}_{5} * \mathrm{BC}_{\mathrm{Max}}-\mathrm{M}_{\mathrm{Na} 2 \mathrm{O}}, 0\right)
$$

Equation 13

igf $f_{6}$

$$
\begin{aligned}
& =\operatorname{IF}\left(\mathrm{BC}_{\text {Flag }}=1, \operatorname{igf}_{6 \mathrm{~b}}, \operatorname{IF}\left(\mathrm{BC}_{\text {Flag }}=6, \operatorname{igf}_{6 \mathrm{~b}}, \operatorname{IF}\left(\mathrm{BC}_{\text {Flag }}=15, \operatorname{IF}\left(\mathrm{M}_{\text {Zro2 }} / \mathrm{BC}_{23}\right) / \mathrm{BC}_{\mathrm{MAX}}>\right.\right.\right. \\
& \left.\left.\left.\left.0.5, \operatorname{igf}_{6 \mathrm{a}}, \operatorname{igf}_{6 \mathrm{~b}}\right), \operatorname{igf}_{6 \mathrm{a}}\right)\right)\right)
\end{aligned}
$$

Equation 14

$$
\mathrm{GF}_{\text {SiO2.p0 }}
$$

$$
\begin{aligned}
& =\operatorname{MAX}\left(\mathrm{BC}_{\mathrm{Max}}-\mathrm{M}_{\text {waste }}-\mathrm{GF}_{\mathrm{AL203.p1}}-\mathrm{GF}_{\mathrm{B} 203 . \mathrm{pl}}-\mathrm{GF}_{\mathrm{Fe} 203 . \mathrm{p0}}-\mathrm{GF}_{\mathrm{Li20.p1}}-\mathrm{GF}_{\mathrm{Na2O} . \mathrm{pl} 1}\right. \text {, } \\
& \mathrm{igf}_{6} *\left(\mathrm{M}_{\text {waste }}+\mathrm{GF}_{\mathrm{Al} 2 \mathrm{O} 3 \mathrm{pl}}+\mathrm{GF}_{\mathrm{B} 2 \mathrm{O} 3 \mathrm{pl}}+\mathrm{GF}_{\mathrm{Fe} 2 \mathrm{O} 3 \mathrm{p} 0}+\mathrm{GF}_{\mathrm{Li2O}, \mathrm{pl}}+\mathrm{GF}_{\mathrm{Na} 2 \mathrm{O} . \mathrm{pl}}\right) /\left(1-\mathrm{igf}_{6}\right) \\
& \left.-\mathrm{M}_{\mathrm{SiO} 2} /\left(1-\mathrm{igf}_{6}\right), 0\right)
\end{aligned}
$$

Equation 15

FracSi

$$
\begin{aligned}
& =\left(\mathrm{GF}_{\mathrm{SiO2.p0}}+\mathrm{M}_{\mathrm{SiO} 2}\right) /\left(\mathrm{M}_{\mathrm{waste}}+\mathrm{GF}_{\mathrm{Al203.p1}}+\mathrm{GF}_{\mathrm{B} 203, \mathrm{p1}}+\mathrm{GF}_{\mathrm{Fe} 203 . \mathrm{p} 0}+\mathrm{GF}_{\mathrm{Li2O.p1}}+\mathrm{GF}_{\mathrm{Na} 20 . \mathrm{p} 1}\right. \\
& \left.+\mathrm{GF}_{\mathrm{SiO2.p0}}\right)
\end{aligned}
$$

Equation 16

$\mathrm{GF}_{\mathrm{Fe} 203, \mathrm{pl}}$

$$
=\mathrm{F}\left(\mathrm{FracSi}>\mathrm{BC}_{18},\left(\mathrm{FracSi}-\mathrm{BC}_{18}\right) * \mathrm{BC}_{\mathrm{Max}}, 0\right)+\mathrm{GF}_{\mathrm{Fe} 203 . \mathrm{p} 0}
$$

Equation 17

$\mathrm{GF}_{\mathrm{SiO}, \mathrm{pl}}$

$$
=\mathrm{IF}\left(\mathrm{FracSi}>\mathrm{BC}_{18},-\left(\mathrm{FracSi}-\mathrm{BC}_{18}\right) * \mathrm{BC}_{\mathrm{Max}}, 0\right)+\mathrm{GF}_{\mathrm{SiO} . \mathrm{p} 0}
$$

\footnotetext{
${ }^{4}$ The validity minimum for Al per PNNL-18501 is 0.019 but the glass shell frequently gives a higher waste loading with a slighter higher minimum as shown in table.

${ }^{5}$ The validity minimum for Si per PNNL-18501 is 0.303 but a slightly higher values generally gives a higher waste loading. Research into glass recipes went down to the 30.3 , and is therefore the validity range, but these low silica glasses are often silica deficient and have problems with the property models that need correction. So, the slightly higher value of 35 wt $\%$ is used. Also an even higher silica value for aluminum, chromium, and phosphate limited glasses frequently gives an even higher waste loading.
} 
Equation 18

$\mathrm{M}_{\mathrm{Glass} . \mathrm{p} 1}=\mathrm{M}_{\mathrm{waste}}+\mathrm{GF}_{\mathrm{Al203.p1}}+\mathrm{GF}_{\mathrm{B} 2 \mathrm{O} 3 \mathrm{p} 1}+\mathrm{GF}_{\mathrm{Fe} 203 . \mathrm{p} 1}+\mathrm{GF}_{\mathrm{Li2O.p1}}+\mathrm{GF}_{\mathrm{Na} 2 \mathrm{O} . \mathrm{p} 1}+\mathrm{GF}_{\mathrm{SiO} 2 \mathrm{p} 1}$

\section{Nepheline Adjustments}

There are two conditions silica must meet. First, there is a minimum amount of silica required to make a glass and second, there is a maximum amount of silica with respect to sodium and aluminum. If too much silica is present, Nepheline (a crystalline mineral) can form that affects durability of glass. To prevent Nepheline precipitation in glasses, Nepheline Rule is used as expressed by Equation 19.

Equation 19

$N_{S i}=\frac{\mathrm{SiO}_{2}}{\mathrm{Al}_{2} \mathrm{O}_{3}+\mathrm{Na}_{2} \mathrm{O}+\mathrm{SiO}_{2}} \geq 0.62$

Solving Equation 19 for $\mathrm{SiO}_{2}$ gives Equation 20.

Equation 20

$\mathrm{SiO}_{2}=\frac{0.62}{1-0.62}\left(\mathrm{Al}_{2} \mathrm{O}_{3}+\mathrm{Na}_{2} \mathrm{O}\right)=\frac{62}{38}\left(\mathrm{Al}_{2} \mathrm{O}_{3}+\mathrm{Na}_{2} \mathrm{O}\right)$

Check to see if the silica additive estimate, $\mathrm{GF}_{\mathrm{SiO} \text {.p1 }}$, needs to be increased and then increase as necessary.

$$
\begin{aligned}
& \mathrm{N}_{\text {nep }} \quad=\left(\mathrm{GF}_{\mathrm{SiO} 2 \mathrm{pl}}+\mathrm{M}_{\mathrm{SiO} 2}\right) /\left(\mathrm{GF}_{\mathrm{SiO} 2 \mathrm{pl}}+\mathrm{M}_{\mathrm{SiO} 2}+\mathrm{GF}_{\mathrm{Al} 2 \mathrm{O} . \mathrm{p} 1}+\mathrm{M}_{\mathrm{Al2O} 3}+\mathrm{GF}_{\mathrm{Na} 2 \mathrm{O} . \mathrm{p} 1}+\mathrm{M}_{\mathrm{Na} 2 \mathrm{O}}\right) \\
& \mathrm{GF}_{\mathrm{SiO} 2 \mathrm{p2a}}=\mathrm{IF}\left(\mathrm{N}_{\text {nep }}<0.62,(62 / 38) *\left(\mathrm{GF}_{\mathrm{Al} 203 . \mathrm{p} 1}+\mathrm{M}_{\mathrm{Al} 2 \mathrm{O} 3}+\mathrm{GF}_{\mathrm{Na} 2 \mathrm{O} . \mathrm{p} 1}+\mathrm{M}_{\mathrm{Na} 2 \mathrm{O}}\right)-\mathrm{M}_{\mathrm{SiO} 2}, \mathrm{GF}_{\mathrm{SiO} 2 \mathrm{p} 1}\right) \\
& \mathrm{M}_{\mathrm{Glass} . \mathrm{p} 2}=\left(\left(\mathrm{GF}_{\mathrm{SiO} 2 \mathrm{p} 2 \mathrm{a}}+\mathrm{M}_{\mathrm{SiO} 2}\right) /\left(\mathrm{GF}_{\mathrm{SiO} 2 \mathrm{p} 1}+\mathrm{M}_{\mathrm{SiO} 2}\right)\right) * \mathrm{M}_{\mathrm{Glass} . \mathrm{p} 1}
\end{aligned}
$$

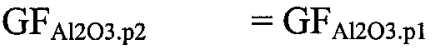

$$
\begin{aligned}
& \mathrm{GF}_{\mathrm{B} 203 . \mathrm{p} 2}=0.30 *\left(\mathrm{M}_{\mathrm{Glass} . \mathrm{p} 2}-\mathrm{M}_{\mathrm{Glass} . \mathrm{p} 1}\right)+\mathrm{GF}_{\mathrm{B} 2 \mathrm{O} \text {.p1 }} \\
& \mathrm{GF}_{\mathrm{Fe} 203 . \mathrm{p} 2}=0.15 *\left(\mathrm{M}_{\mathrm{Glass} . \mathrm{p} 2}-\mathrm{M}_{\mathrm{Glass} . \mathrm{p} 1}\right)+\mathrm{GF}_{\mathrm{Fe} 203 . \mathrm{p} 1} \\
& \mathrm{GF}_{\mathrm{Li2O}, \mathrm{p} 2}=0.06 *\left(\mathrm{M}_{\mathrm{Glass} . \mathrm{p} 2}-\mathrm{M}_{\mathrm{Glass} . \mathrm{pl}}\right)+\mathrm{GF}_{\mathrm{Li2O} . \mathrm{p} 1} \\
& \mathrm{GF}_{\mathrm{Na} 2 \mathrm{O} . \mathrm{p2}}=\mathrm{GF}_{\mathrm{Na} 2 \mathrm{O} . \mathrm{p} 1}
\end{aligned}
$$

Any room left in the glass shall be taken by the $\mathrm{SiO}_{2}$ glass former. Therefore, $\mathrm{GF}_{\mathrm{SiO2} \text {.p2a }}$ may be increased. The result is $\mathrm{GF}_{\mathrm{SiO} 2 . \mathrm{p2}}$.

$$
\begin{array}{ll}
\mathrm{GF}_{\mathrm{SiO2} . \mathrm{p} 2} & =\mathrm{MAX}\left(\mathrm{M}_{\mathrm{Glass} . \mathrm{p} 2}-\mathrm{GF}_{\mathrm{A} 1203 . \mathrm{p} 2}-\mathrm{GF}_{\mathrm{B} 203 . \mathrm{p} 2}-\mathrm{GF}_{\mathrm{Fe} 2 \mathrm{O}, \mathrm{p} 2}-\mathrm{GF}_{\mathrm{Li2} \text {. p2 }}-\mathrm{GF}_{\mathrm{Na} 2 \mathrm{O} . \mathrm{p} 2}-\mathrm{M}_{\mathrm{waste}}, 0.0\right) \\
\text { GlassProp }_{1} & =1
\end{array}
$$

\section{One-Percent Crystal $\left(\mathrm{T}_{1 \%}\right)$ or Spinel-Limited Glass Adjustment}

The One-Percent Crystal ( $\mathrm{T}_{1 \%}$, Equation 21$)$, which is a Liquidus Temperature model, often has the biggest impact on the quantity of glass made. Molten glass that cools to $950^{\circ} \mathrm{C}$ and forms one percent or less of spinel crystals is desired. The amount of spinel crystals is largely dependent on the type of waste 
in the glass. The waste constituents that have the biggest impact on $\mathrm{T}_{1 \%}$ are listed in Table 4 . If the glass has a high $T_{1 \%}$ value, swapping glass former to bring down the $T_{1 \%}$ value does not help much. Swapping glass formers only has merit in fine-tuning the recipe and quickly becomes too tedious for mission studies. Therefore, this routine will adjust $T_{1 \%}$ by diluting the PreGlass $\left(\mathrm{M}_{\mathrm{Glass} . \mathrm{p} 2}\right)$ with a low $\mathrm{T}_{1 \%}$ value glass shown in Table 5 and Table 6 . If the waste contains a significant amount of aluminum, Dilution Glass A (Table 5) shall be used. If the waste contains low amounts of aluminum or iron, Dilution Glass B (Table 6) shall be used. The routine for dilution follows. The first part of the routine is to determine if dilution is even necessary.

$$
\begin{aligned}
& \mathrm{NM} \text { (noble metals) }=\mathrm{M}_{\mathrm{PdO}}+\mathrm{M}_{\mathrm{Rh} 203}+\mathrm{M}_{\mathrm{RuO2}} \\
& \mathrm{M}_{\mathrm{others} \mathrm{T} 1 \%}=\mathrm{M}_{\mathrm{others}}-\mathrm{M}_{\mathrm{Cr} 2 \mathrm{O} 3}-\mathrm{M}_{\mathrm{K} 2 \mathrm{O}}-\mathrm{M}_{\mathrm{MgO}}-\mathrm{M}_{\mathrm{MnO}}-\mathrm{M}_{\mathrm{NiO}}-\mathrm{M}_{\mathrm{PdO}}-\mathrm{M}_{\mathrm{Rh} 2 \mathrm{O} 3}-\mathrm{M}_{\mathrm{RuO2} 2}-\mathrm{M}_{\mathrm{SrO}}-\mathrm{M}_{\mathrm{ThO} 2} \\
& \text { - } \mathrm{M}_{\mathrm{ZnO}}-\mathrm{M}_{\mathrm{ZrO} 2}
\end{aligned}
$$

Equation 21

$$
T_{1 \% \text {. p } 2}=\sum_{i=1}^{16} \mathrm{~b}_{\mathrm{i}} \frac{M_{i}+G F_{i}}{M_{\text {Glass. } p 2}}+b_{17} \frac{M_{\text {others } T 1 \%}}{M_{\text {Glass. } p 2}}
$$

where: $\quad \mathrm{T}_{1 \% \mathrm{p} 2}$ is the Spinel Temperature $\left(\mathrm{T}_{1 \%}\right)$ for PreGlass $\left(\mathrm{M}_{\mathrm{Glass} . \mathrm{p} 2}\right)$.

$i$ is the $i^{\text {th }}$ component in Table 4 .

$\mathrm{M}_{\mathrm{i}}$ is the Mass of $i$-th waste component listed in Table 4 . There are 16 components plus Others.

$\mathrm{GF}_{\mathrm{i}}$ is the Mass of the $i$-th glass former component $\left(\mathrm{GF}_{\mathrm{ip} 2}\right)$ matching the waste component listed in Table 4.

$b_{i}$ is the component coefficient for $i$-th component in Table 4 .

IF $\mathrm{T}_{1 \%}>950$ THEN GOTO Dilution Subroutine

ELSE

$$
\begin{aligned}
& \mathrm{M}_{\text {Glass.p3 }}=\mathrm{M}_{\text {Glass.p2 }} \\
& \mathrm{GF}_{\mathrm{Al} 203 . \mathrm{p} 3}=\mathrm{GF}_{\mathrm{Al} 203 . \mathrm{p} 2} \\
& \mathrm{GF}_{\mathrm{B} 2 \mathrm{O} 3 \mathrm{p} 3}=\mathrm{GF}_{\mathrm{B} 2 \mathrm{O} 3 \mathrm{p2}} \\
& \mathrm{GF}_{\mathrm{Fe} 203 . \mathrm{p} 3}=\mathrm{GF}_{\mathrm{Fe} 203 . \mathrm{p} 2} \\
& \mathrm{GF}_{\mathrm{Li} 2 \mathrm{O}, \mathrm{p} 3}=\mathrm{GF}_{\mathrm{Li20.p2}} \\
& \mathrm{GF}_{\mathrm{Na} 2 \mathrm{O}, \mathrm{p} 3}=\mathrm{GF}_{\mathrm{Na} 20 . \mathrm{p} 2} \\
& \mathrm{GF}_{\mathrm{SiO} 2, \mathrm{p} \text { 3 }} \quad=\mathrm{GF}_{\mathrm{SiO} 2 \mathrm{p} 2} \\
& \mathrm{~T}_{1 \% \mathrm{p} 3}=\mathrm{T}_{1 \% \mathrm{p} 2}
\end{aligned}
$$

GOTO Glass Viscosity Routine

END IF 


\section{Glass Dilution Subroutine}

The $\mathrm{T}_{1 \%}$ model is a linear fit. Therefore, the mass of Dilution Glass $\left(\mathrm{M}_{\mathrm{DG}}\right)$ can be calculated using a weighted-average temperature impact of the Dilution Glass $\left(\mathrm{M}_{\mathrm{DG}}\right)$ on the PreGlass $\left(\mathrm{M}_{\mathrm{Glass} . \mathrm{p} 2}\right)$.

Equation 22

$T_{1 \%, p 3}=\frac{T_{1 \% . p 2} M_{\text {Glass. } 22}+T_{1 \% D G} M_{D G}}{M_{\text {Glass.p2 }}+M_{D G}}$

Solving Equation 22 for the mass of Dilution Glass $\left(\mathrm{M}_{\mathrm{DG}}\right)$ gives Equation 23.

Equation 23

$M_{D G}=\frac{T_{1 \%, p 2}-T_{1 \%, p 3}}{T_{1 \%, p 3}-T_{1 \%, D G}} M_{G l a s s . p 2} \quad\left(\right.$ Desired $T_{1 \%, p 3}$ is $\left.950^{\circ} \mathrm{C}\right)$

where: $\mathrm{T}_{1 \% \mathrm{DG}}$ is the spinel $\mathrm{T}_{1 \%}$ temperature of the dilution glass being used.

If the PreGlass $\left(\mathrm{M}_{\mathrm{Glass}, \mathrm{p} 2}\right)$ contains a significant amount of aluminum or iron, it is best to use a dilution glass that contains no aluminum or iron. Table 5 shows a dilution glass with no aluminum or iron. It has $\mathrm{T}_{1 \% \mathrm{DG}}$ temperature of $69^{\circ} \mathrm{C}$. Table 6 shows a dilution glass with aluminum and iron with a $\mathrm{T}_{1 \% \mathrm{DG}}$ of 334 ${ }^{\circ} \mathrm{C}$. Logic for determining which dilution glass to use and its amount follows.

$\operatorname{MaxAlFe}=\operatorname{MAX}\left(\left(\mathrm{M}_{\mathrm{Al} 203}+\mathrm{GF}_{\mathrm{Al} 203 . \mathrm{p} 2}\right) /\left(\mathrm{M}_{\mathrm{Glass} . \mathrm{p} 2} * \mathrm{BC}_{1}\right),\left(\mathrm{M}_{\mathrm{Fe} 2 \mathrm{O} 3}+\mathrm{GF}_{\mathrm{Fe} 203 . \mathrm{p} 2}\right) /\left(\mathrm{M}_{\mathrm{Glass} . \mathrm{p} 2} * \mathrm{BC}_{8}\right)\right)$

IF $\mathrm{MaxAlFe}>0.8$

THEN

$\begin{array}{ll}\mathrm{DG}_{\mathrm{A} 2 \mathrm{O} 3} & =0.0 \\ \mathrm{DG}_{\mathrm{B} 2 \mathrm{O} 3} & =0.13 \\ \mathrm{DG}_{\mathrm{Fe} 203} & =0.0 \\ \mathrm{DG}_{\mathrm{Li2O}} & =0.02 \\ \mathrm{DG}_{\mathrm{Na} 2 \mathrm{O}} & =0.22 \\ \mathrm{DG}_{\mathrm{SiO} 2} & =0.63 \\ \mathrm{~T}_{1 \% \mathrm{DG}} & =69 \\ \text { GlassProp } & =2\end{array}$

ELSE

$\begin{array}{ll}\mathrm{DG}_{\mathrm{Al} 2 \mathrm{O} 3} & =0.03 \\ \mathrm{DG}_{\mathrm{B} 2 \mathrm{O} 3} & =0.10 \\ \mathrm{DG}_{\mathrm{Fe} 2 \mathrm{O} 3} & =0.06 \\ \mathrm{DG}_{\mathrm{Li} 2 \mathrm{O}} & =0.02 \\ \mathrm{DG}_{\mathrm{Na} 2 \mathrm{O}} & =0.22 \\ \mathrm{DG}_{\mathrm{SiO} 2} & =0.57 \\ \mathrm{~T}_{1 \% \mathrm{DG}} & =334 \\ \text { GlassProp }_{3} & =3\end{array}$

END IF 


$$
\begin{aligned}
& \mathrm{M}_{\mathrm{DG}} \quad=\left(\mathrm{T}_{1 \% \mathrm{p} 2}-950\right) /\left(950-\mathrm{T}_{1 \% \mathrm{DG}}\right) * \mathrm{M}_{\mathrm{Glass} \mathrm{p} 2} \\
& \mathrm{M}_{\text {Glass.p3 }}=\mathrm{M}_{\mathrm{DG}}+\mathrm{M}_{\text {Glass.p2 }} \\
& \mathrm{GF}_{\mathrm{Al} 203 . \mathrm{p} 3} \quad=\mathrm{DG}_{\mathrm{Al} 203} * \mathrm{M}_{\mathrm{DG}}+\mathrm{GF}_{\mathrm{Al} 203, \mathrm{p} 2} \\
& \mathrm{GF}_{\mathrm{B} 203 . \mathrm{p} 3}=\mathrm{DG}_{\mathrm{B} 2 \mathrm{O} 3} * \mathrm{M}_{\mathrm{DG}}+\mathrm{GF}_{\mathrm{B} 2 \mathrm{O} 3 \mathrm{p} 2} \\
& \mathrm{GF}_{\mathrm{Fe} 2 \mathrm{O} 3 \mathrm{p} 3}=\mathrm{DG}_{\mathrm{Fe} 2 \mathrm{O} 3} * \mathrm{M}_{\mathrm{DG}}+\mathrm{GF}_{\mathrm{Fe} 203, \mathrm{p} 2} \\
& \mathrm{GF}_{\mathrm{Li20.p3}}=\mathrm{DG}_{\mathrm{Li20}} * \mathrm{M}_{\mathrm{DG}}+\mathrm{GF}_{\mathrm{Li2O} \text {. } 2} \\
& \mathrm{GF}_{\mathrm{Na} 2 \mathrm{O} . \mathrm{p} 3}=\mathrm{DG}_{\mathrm{Na} 2 \mathrm{O}} * \mathrm{M}_{\mathrm{DG}}+\mathrm{GF}_{\mathrm{Na} 20 . \mathrm{p} 2} \\
& \mathrm{GF}_{\mathrm{SiO} 2 \mathrm{p} 3} \quad=\mathrm{DG}_{\mathrm{SiO} 2} * \mathrm{M}_{\mathrm{DG}}+\mathrm{GF}_{\mathrm{SiO} 2 \mathrm{p} 2} \\
& \mathrm{~T}_{1 \% \mathrm{p} 3}=950
\end{aligned}
$$

\begin{tabular}{|c|c|c|}
\hline (i) & $g_{i}$ & Coefficient $\left(b_{i}\right)$ \\
\hline \multicolumn{3}{|c|}{ Predominantly Waste Oxides } \\
\hline 1 & $\mathrm{Al}_{2} \mathrm{O}_{3}$ & 2,835 \\
\hline 2 & $\mathrm{Cr}_{2} \mathrm{O}_{3}$ & 12,468 \\
\hline 3 & $\mathrm{Fe}_{2} \mathrm{O}_{3}$ & 3,328 \\
\hline 4 & $\mathrm{~K}_{2} \mathrm{O}$ & -410 \\
\hline 5 & $\mathrm{MgO}$ & 3,927 \\
\hline 6 & $\mathrm{MnO}$ & 2,619 \\
\hline 7 & $\mathrm{NiO}$ & 11,916 \\
\hline 8 & SrO & 421 \\
\hline 9 & $\mathrm{ThO}_{2}$ & 897 \\
\hline 10 & $\mathrm{ZnO}$ & 3,103 \\
\hline 11 & $\mathrm{ZrO}_{2}$ & 1,933 \\
\hline 12 & NM & 14,871 \\
\hline \multicolumn{3}{|c|}{ Predominantly GF Oxides } \\
\hline 13 & $\mathrm{~B}_{2} \mathrm{O}_{3}$ & -201 \\
\hline 14 & $\mathrm{Li}_{2} \mathrm{O}$ & -735 \\
\hline 15 & $\mathrm{Na}_{2} \mathrm{O}$ & -718 \\
\hline 16 & $\mathrm{SiO}_{2}$ & 425 \\
\hline 17 & Others T1\% & 736 \\
\hline
\end{tabular}

Table $4 \quad T_{1 \%}{ }^{\circ} \mathrm{C} / \mathrm{w} \%$ Model Coefricients

Table 5 Dilution Glass A

\begin{tabular}{|l|l|l|r|r|r|}
\hline GF/Oxide & wt\% & Property & Value & Minimum & Maximum \\
\hline $\mathrm{Al}_{2} \mathrm{O}_{3}$ & 0 & $\mathrm{~T}_{1 \%}-\mathrm{Sp},{ }^{\circ} \mathrm{C}$ & 69 & & 950 \\
\hline $\mathrm{B}_{2} \mathrm{O}_{3}$ & 13 & $\mathrm{~T}_{\mathrm{L}}{ }^{\circ} \mathrm{C}$ & 411 & 1050 \\
\hline $\mathrm{Fe}_{2} \mathrm{O}_{3}$ & 0 & Visc1 $150, \mathrm{~Pa} . \mathrm{s}$ & 4.25 & 4 & \\
\hline $\mathrm{Li}_{2} \mathrm{O}$ & 2 & Nepheline, $\mathrm{N}_{\mathrm{Si}}$ & $74.1 \%$ & $62 \%$ & \\
\hline $\mathrm{Na}_{2} \mathrm{O}$ & 22 & & & & \\
\hline $\mathrm{SiO}_{2}$ & 63 & & & & \\
\hline
\end{tabular}


Table 6 Diution Glass B (With iron and aluminum)

\begin{tabular}{|l|l|l|r|r|r|}
\hline GF/Oxide & wt\% & Property & Value & Minimum & Maximum \\
\hline $\mathrm{Al}_{2} \mathrm{O}_{3}$ & 3 & $\mathrm{~T}_{1 \%}-\mathrm{Sp},{ }^{\circ} \mathrm{C}$ & 334 & & 950 \\
\hline $\mathrm{B}_{2} \mathrm{O}_{3}$ & 10 & $\mathrm{~T}_{\mathrm{L}},{ }^{\circ} \mathrm{C}$ & 488 & & 1050 \\
\hline $\mathrm{Fe}_{2} \mathrm{O}_{3}$ & 6 & Visc1150, Pa.s & 4.97 & 4 & \\
\hline $\mathrm{Li}_{2} \mathrm{O}$ & 2 & Nepheline, $\mathrm{N}_{\mathrm{Si}}$ & $69.5 \%$ & $62 \%$ & \\
\hline $\mathrm{Na}_{2} \mathrm{O}$ & 22 & & & & \\
\hline $\mathrm{SiO}_{2}$ & 57 & & & & \\
\hline
\end{tabular}

\section{Glass Viscosity}

Viscosity of the PreGlass can be determined by the following model equation, which is a curve fit of numerous glasses with varying compositions.

Equation 24

$$
\ln \left(\eta_{G, p 3}\right)=\sum_{i=1}^{19} c_{i} g_{i, p 3}+c_{20} g_{\text {Na2O.p3 }} g_{B 2 O 3, p 3}+c_{21} g_{\text {Na2O.p3 }} g_{A l 2 O 3, p 3}+c_{22} g_{B 2 O 3 . p 3}^{2}+c_{23} g_{C a O, p 3} g_{A l 2 O 3 . p 3}+c_{24} g_{L i 2 O, p 3}^{2}
$$

where: $\quad \ln (\eta)$ is the natural log of the glass viscosity $\eta . \eta$ is in Pascal-seconds (Pa-s). The subscript G.p3 means the viscosity is for the PreGlass $\left(M_{G \text { lass.p } 3}\right)$.

$i$ is the $i^{\text {th }}$ component in Table 7 .

$\mathrm{g}_{i}$ is the mass fraction of the component in the PreGlass $\left(\mathrm{M}_{\mathrm{Glass} . \mathrm{p} 3}\right)$, i.e., $\mathrm{g}_{11}=\left(\mathrm{GF}_{\mathrm{Na2O.p3}}+\right.$ $\left.\mathrm{M}_{\mathrm{Na} 2 \mathrm{O}}\right) / \mathrm{M}_{\mathrm{Glass} . \mathrm{p} 3}$

$c_{i}$ is the coefficient of the $i^{\text {th }}$ component in Table 7.

$$
\begin{aligned}
M_{\text {others }}= & M_{\text {others }}+M_{\mathrm{Fe} 203}-M_{\mathrm{BaO}}-M_{\mathrm{CaO}}-M_{\mathrm{F}}-M_{\mathrm{K} 2 \mathrm{O}}-M_{\mathrm{La} 203}-M_{\mathrm{MgO}}-M_{\mathrm{MnO}}-M_{\mathrm{P} 2 \mathrm{O} 5}-\mathrm{M}_{\mathrm{PbO}} \\
& -\mathrm{M}_{\mathrm{SrO}}-M_{\mathrm{UO} 3}-\mathrm{M}_{\mathrm{ZnO}}-\mathrm{M}_{\mathrm{ZrO2}}
\end{aligned}
$$

The glass viscosity shall be adjusted (if necessary) to be at or between 4 and $6 \mathrm{~Pa}$-s. Fitting to the natural $\log$ takes some of the non-linearity out of the programming logic. Therefore the glass will actually be adjusted to be at or between $\ln (4 \mathrm{~Pa}-\mathrm{s})$ and $\ln (6 \mathrm{Pas}-\mathrm{s})$. 
Table 7 In[muso] Model Coefficients

\begin{tabular}{|c|c|c|}
\hline (i) & $\mathbf{g}_{i}$ & Coefficient $\left(c_{i}\right)$ \\
\hline 1 & $\mathrm{Al}_{2} \mathrm{O}_{3}$ & 10.60850109 \\
\hline 2 & $\mathrm{~B}_{2} \mathrm{O}_{3}$ & -9.375292157 \\
\hline 3 & $\mathrm{BaO}$ & -3.418158325 \\
\hline 4 & $\mathrm{CaO}$ & -6.932801478 \\
\hline 5 & $F$ & -12.34448744 \\
\hline 6 & $\mathrm{~K}_{2} \mathrm{O}$ & -3.824906136 \\
\hline 7 & $\mathrm{La}_{2} \mathrm{O}_{3}$ & -4.969542588 \\
\hline 8 & $\mathrm{Li}_{2} \mathrm{O}$ & -39.02491029 \\
\hline 9 & $\mathrm{MgO}$ & -3.231407331 \\
\hline 10 & $\mathrm{MnO}$ & -6.886774142 \\
\hline 11 & $\mathrm{Na}_{2} \mathrm{O}$ & -9.632750972 \\
\hline 12 & $\mathrm{P}_{2} \mathrm{O}_{5}$ & 5.305006777 \\
\hline 13 & $\mathrm{PbO}$ & -23.14362222 \\
\hline 14 & $\mathrm{SiO}_{2}$ & 9.368088941 \\
\hline 15 & $\mathrm{SrO}$ & -4.350515691 \\
\hline 16 & $\mathrm{UO}_{3}$ & 2.151455253 \\
\hline 17 & $\mathrm{ZnO}$ & -2.696255253 \\
\hline 18 & $\mathrm{ZrO}_{2}$ & 7.140440337 \\
\hline 19 & Othersn & -0.090267559 \\
\hline 20 & $\mathrm{Na}_{2} \mathrm{O} \times \mathrm{B}_{2} \mathrm{O}_{3}$ & -26.95708996 \\
\hline 21 & $\mathrm{Na}_{2} \mathrm{O} \times \mathrm{Al}_{2} \mathrm{O}_{3}$ & 17.5171818 \\
\hline 22 & $\mathrm{~B}_{2} \mathrm{O}_{3} \times \mathrm{B}_{2} \mathrm{O}_{3}$ & 24.5926202 \\
\hline 23 & $\mathrm{CaO} \times \mathrm{Al}_{2} \mathrm{O}_{3}$ & -8.134744021 \\
\hline 24 & $\mathrm{Li}_{2} \mathrm{O} \times \mathrm{Li}_{2} \mathrm{O}$ & 47.35918258 \\
\hline
\end{tabular}

IF $\ln (6) \geq \ln \left(\eta_{\mathrm{G.p} 3}\right) \geq \ln (4)$ THEN

(a IF THEN)

$\ln \left(\eta_{\text {G.p5 }}\right)=\ln \left(\eta_{\text {G.p3 }}\right)$

$\mathrm{M}_{\mathrm{Glass} . \mathrm{p} 5} \quad=\mathrm{M}_{\mathrm{Glass} . \mathrm{p3}}$

$\mathrm{GF}_{\mathrm{Al203,p5}}=\mathrm{GF}_{\mathrm{Al203.p3}}$

$\mathrm{GF}_{\mathrm{B} 203, \mathrm{p} 5}=\mathrm{GF}_{\mathrm{B} 203, \mathrm{p3}}$

$\mathrm{GF}_{\mathrm{Fe} 203, \mathrm{p} 5}=\mathrm{GF}_{\mathrm{Fe} 203, \mathrm{p} 3}$

$\mathrm{GF}_{\mathrm{Li20.p5}} \quad=\mathrm{GF}_{\mathrm{Li2O}, \mathrm{p} 3}$

$\mathrm{GF}_{\mathrm{Na20} . \mathrm{ps}}=\mathrm{GF}_{\mathrm{Na} 20 . \mathrm{p3}}$

$\mathrm{GF}_{\mathrm{SiO} 2 \mathrm{ps}} \quad=\mathrm{GF}_{\mathrm{SiO2} \text {. } 33}$

GOTO Product Consistency Test

ELSE

(a ELSE)

IF $\ln \left(\eta_{\mathrm{G} . \mathrm{p} 3}\right)<\ln (4) \mathrm{THEN}$

(b IF THEN)

GOTO Low Viscosity Subroutine

ELSE

(b ELSE) 
GOTO High Viscosity Subroutine

END IF

(b END)

END IF

(a END)

\section{$\underline{\text { High Viscosity Adjustment }}$}

If the viscosity of the PreGlass $\left(\eta_{\mathrm{G} . \mathrm{p} 3}\right)$ from Equation 24 is higher than the desired highest viscosity $\left(\eta_{\mathrm{h}}\right)$, then the viscosity of the PreGlass needs to be lowered to the desired highest viscosity $\left(\eta_{h}\right)$. The additive $\mathrm{Li}_{2} \mathrm{O}$ will be exchanged for the additive $\mathrm{B}_{2} \mathrm{O}_{3}$, kilogram for kilogram, until $\eta_{\mathrm{G} . p 3}$ equals $\eta_{\mathrm{h}}$. The terms that are affected in general viscosity equation (Equation 24) are given in Equation 25. How many kilograms need to be swapped can be derived from the logic that follows. Exchanging $\mathrm{Li}_{2} \mathrm{O}$ for $\mathrm{B}_{2} \mathrm{O}_{3}$ will reduce the viscosity because $\mathrm{Li}_{2} \mathrm{O}$ 's coefficients are more negative than $\mathrm{B}_{2} \mathrm{O}_{3}$ 's coefficients. Either $\mathrm{Li}_{2} \mathrm{O}$ or $\mathrm{B}_{2} \mathrm{O}_{3}$ can represent the mass change since they are being exchanged kilogram for kilogram. However, this unknown is not easily solved directly. $\Delta_{\mathrm{LizO}}$ will be determined numerically by using the Newton's Method ${ }^{6}$.

Equation 25

$\ln \left(\eta_{L i B}\right)=c_{2} g_{B 2 O 3 . p 3}+c_{8} g_{L i 2 O . p 3}+c_{20} g_{N a 2 O . p 3} g_{B 2 O 3 . p 3}+c_{22} g_{B 2 O 3, p 3}^{2}+c_{24} g_{L i 2 O . p 3}^{2}$

where: $\quad c_{i}$ coefficients come from Table 7.

The viscosity equation (Equation 24) can now be represented by two main variables. The part that is affected by the $\mathrm{Li}_{2} \mathrm{O}$ swap, $\ln \left(\eta_{L i B}\right)$, and that which is not $\ln \left(\eta_{\text {NonLiB }}\right)$.

Equation 26

$\ln \left(\eta_{G, p 3}\right)=\ln \left(\eta_{L i B}\right)+\ln \left(\eta_{\text {NonLiBI }}\right)$

How the desired high viscosity $\left(\eta_{h}\right)$ relates is given in Equation 27.

Equation 27

$\ln \left(\eta_{h}\right)=\ln \left(\eta_{L i B}\right)+\ln \left(\eta_{\Delta L i B}\right)+\ln \left(\eta_{\text {NonLiBI }}\right)$

where: $\quad \eta_{\mathrm{h}}$ is the desired highest viscosity, which is $6 \mathrm{~Pa}$-s that gives a $\ln \left(\eta_{h}\right)$ value of 1.79176 .

$\eta_{\Delta \mathrm{LiB}}$ is the desired drop in the viscosity from $\eta_{\mathrm{G} . \mathrm{p} 3}$ to $\eta_{\mathrm{h}}$.

Substituting $\ln \left(\eta_{\text {NonLiBI }}\right)$ from Equation 26 into Equation 27 gives Equation 28.

Equation 28

$\ln \left(\eta_{h}\right)=\ln \left(\eta_{L i B}\right)+\ln \left(\eta_{\Delta L i B}\right)+\ln \left(\eta_{G . p 3}\right)-\ln \left(\eta_{L i B}\right)$ or

${ }^{6}$ Also known as the Newton-Raphson Method, named after Issac Newton and Joseph Raphson. Given the function $f(x)$ and its derivative $f^{\prime}(x)$, we begin with a first guess or approximation $x_{0}$.

A better approximation $x_{1}$ is: $\quad x_{1}=x_{0}-\frac{f\left(x_{0}\right)}{f^{\prime}\left(x_{0}\right)}$. 


$$
\ln \left(\eta_{\Delta x i B}\right)=\ln \left(\eta_{h}\right)-\ln \left(\eta_{G, p 3}\right)
$$

The viscosity is lowered by adding $\mathrm{Li}_{2} \mathrm{O}$. To keep the waste loading of the glass constant, $\mathrm{Li}_{2} \mathrm{O}$ is swapped $\mathrm{kg}$-per-kg of $\mathrm{B}_{2} \mathrm{O}_{3}$ as shown in Equation 29.

Equation 29

$\ln \left(\eta_{\Delta L i B}\right)=c_{2}\left(g_{B 2 O 3, p 3}-\Delta_{B 2 O 3}\right)-c_{2} g_{B 2 O 3, p 3}+c_{8}\left(g_{L i 2 O . p 3}+\Delta_{L i 2 O}\right)-c_{8} g_{L i 2 O, p 3}+c_{20} g_{N a 2 O, p 3}\left(g_{B 2 O 3 . p 3}-\Delta_{B 2 O 3}\right)-c_{20} g_{N a 20 . p 3} g_{B 203 . p 3}$ $+c_{22}\left(g_{B 2 O 3 . p 3}-\Delta_{B 2 O 3}\right)^{2}-c_{22} g_{B 2 O O_{1, p 3}}^{2}+c_{24}\left(g_{L i 2 O, p 3}+\Delta_{L i 2 O}\right)^{2}-c_{24} g_{L i 2 O, p 3}^{2}$

$\mathrm{Li}_{2} \mathrm{O}$ can be used to represent both $\mathrm{Li}_{2} \mathrm{O}$ and $\mathrm{B}_{2} \mathrm{O}_{3}$ since they are the same amount but one is the negative of the other as shown in Equation 29. Substituting terms for the exchange of some $\mathrm{LiO}_{2}$ for $\mathrm{B}_{2} \mathrm{O}_{3}$ from Equation 30 in Equation 29 gives Equation 31 with one unknown $\left(\Delta_{\mathrm{I} i 20}\right)$. This is the equation used in the subroutine to determine the amount of $\mathrm{Li}_{2} \mathrm{O}$ to swap with $\mathrm{B}_{2} \mathrm{O}_{3}$.

Equation 30

$\Delta_{L i 2 O}=\Delta_{B 2 O 3}=\Delta$ for shorthand. (units wt fraction)

Equation 31

$\ln \left(\eta_{\Delta}\right)=-c_{2} \Delta+c_{8} \Delta-c_{20} g_{N a 2 O_{, p 3}} \Delta+c_{22}\left|\left(g_{B 203 . p 3}-\Delta\right)^{2}-g_{B 2 O 3, p 3}^{2}\right|+c_{24}\left|\left(g_{L i 2 O, p^{3}}+\Delta\right)^{2}-g_{L i 20 . p 3}^{2}\right|$

\section{High Viscosity Subroutine}

$\Delta_{0}=\operatorname{MAX}\left(0.01, \mathrm{~g}\left(\mathrm{GF}_{\mathrm{Li} \mathrm{i} O \mathrm{p} 33}+\mathrm{M}_{\mathrm{Li2O}}\right) / \mathrm{M}_{\mathrm{Glass} . \mathrm{p} 3}\right)$ or $\mathrm{MIN}\left(0.01, \mathrm{~g}_{\mathrm{Li} 2 \mathrm{O} . \mathrm{p} 3}\right)$

$\mathrm{DO} n=0$ to 5

$$
\begin{aligned}
& f\left(\Delta_{n}\right)=c_{2} \Delta_{n}-c_{8} \Delta_{n}+c_{20} g_{N a 2 O ., 33} \Delta_{n}+c_{22}\left[g_{B 2 O 3 . p 3}^{2}-\left(g_{B 2 O 3 . p 3}-\Delta_{n}\right)^{2}\right]+c_{24}\left[g_{L i 2 O . p 3}^{2}-\left(g_{L i 2 O . p 3}+\left.\Delta_{n}\right|^{2}\right]\right. \\
& -\left(\ln \left(\eta_{G . p .3}\right)-\ln \left(\eta_{h}\right)\right) \\
& f^{\prime}\left(\Delta_{n}\right)=c_{2}-c_{8}+c_{20} g_{N a 2 O . p 3}+2 c_{22}\left(g_{B 2 O 3 . p 3}-\Delta_{n}\right)-2 c_{24}\left(g_{L i 2 O . p 3}+\Delta_{n}\right)
\end{aligned}
$$

NEXT $n$

END DO

$\Delta_{\mathrm{Li}}=\Delta_{\mathrm{n}=6}$

IF $\mathrm{gLi}_{\mathrm{Li} 2 \mathrm{p} 3}+\Delta_{\mathrm{L}, \mathrm{j}}>0.06$ (i.e., $6.0 \mathrm{wt} \% \mathrm{Li}_{2} \mathrm{O}$ in glass) $\mathrm{THEN}$

GOTO High Viscosity Dilution Subroutine 


\section{ELSE}

$\mathrm{M}_{\mathrm{Glass} .5}=\mathrm{M}_{\mathrm{Glass.p3}}$

\begin{tabular}{|c|c|}
\hline $\mathrm{GF}_{\mathrm{A} 1203.5}$ & $=\mathrm{GF}_{\mathrm{A} 1203 . \mathrm{p} 3}$ \\
\hline $\mathrm{GF}_{\mathrm{B} 203.5}$ & $=\mathrm{GF}_{\mathrm{B} 203 . \mathrm{p} 3}-\Delta_{\mathrm{Li}} * \mathrm{M}_{\mathrm{Glass.p3}}$ \\
\hline $\mathrm{GF}_{\mathrm{Fe} 203.5}$ & $=\mathrm{GF}_{\mathrm{Fe} 203, \mathrm{p} 3}$ \\
\hline $\mathrm{GF}_{\mathrm{Li2O.5}}$ & $=\mathrm{GF}_{\mathrm{Li} 2 \mathrm{p}, \mathrm{p} 3}+\Delta_{\mathrm{Li}} * \mathrm{M}_{\mathrm{Glass}, \mathrm{p} 3}$ \\
\hline $\mathrm{GF}_{\mathrm{Na} 20.5}$ & $=\mathrm{GF}_{\mathrm{Na} 2 \mathrm{O} . \mathrm{p} 3}$ \\
\hline $\mathrm{GF}_{\mathrm{SiO} 2.5}$ & $=\mathrm{GF}_{\mathrm{SiO} 2 \mathrm{p} 3}$ \\
\hline $\ln \left(\eta_{G . p 5}\right)$ & $=\ln (6)$ \\
\hline GlassProp $_{4}$ & $=4$ \\
\hline
\end{tabular}

GOTO Product Consistency Test

\section{High Viscosity Dilution Subroutine}

No more lithium can be placed in $\mathrm{M}_{\mathrm{Glass.p3}}$ if the above routine cannot make the required adjustment. Therefore, this glass needs to be diluted with another glass to further reduce the viscosity and make the final viscosity adjustment. Before doing this, however, the viscosity of the new $\mathbf{M}_{\text {Glass.p } 3}$ with maximum lithium (which is the $\mathrm{M}_{\text {Glass.p4 }}$ ) needs to be calculated. Equation 32 shows how much lithium to use in Equation 33. Equation 33 is an adaptation of Equation 31, which calculates the viscosity adjustment from the $\mathrm{Li}_{2} \mathrm{O}-\mathrm{B}_{2} \mathrm{O}_{3}$ swap.

Equation 32

$\Delta_{L i 2 O X}=0.06-g_{L i 2 O . p 3}$

Equation 33

$$
\ln \left(\eta_{, p 4}\right)=\ln \left(\eta_{, p 3}\right)-\left[\begin{array}{l}
c_{2} \Delta_{L i 2 O X}-c_{8} \Delta_{L i 2 O X}+c_{20} g_{N a 2 O, p 3} \Delta_{L i 2 O X} \\
+c_{22}\left[g_{B 2 O 3, p 3}^{2}-\left(g_{B 2 O 3, p 3}-\Delta_{L i 2 O X}\right)^{2}\right] \\
+c_{24}\left[g_{L i 2 O, p 3}^{2}-\left(g_{L i 2 O, p 3}+\Delta_{L i 2 O X}\right)^{2}\right]
\end{array}\right]
$$

Like the dilution glass routine, if Glass.p4 has a higher content of aluminum it is best to reduce the viscosity of Glass.p4 with a low viscosity dilution glass without aluminum as shown in Table 8 . If Glass.p4 has a lower content of aluminum it is best to reduce the viscosity of Glass.p4 with a low viscosity dilution glass that has aluminum as shown in Table 9 . Since the viscosity model is nonlinear, this routine is not exact but should provide a close estimate. It provides glass quantities sufficient for estimating WTP mission values. 
Table 8 Low Viscosity Glass

\begin{tabular}{|l|l|l|r|r|r|}
\hline GF/Oxide & wt $\%$ & Property & Value & Minimum & Maximum \\
\hline $\mathrm{Al}_{2} \mathrm{O}_{3}$ & 0 & $\mathrm{~T}_{1 \%}-\mathrm{Sp},{ }^{\circ} \mathrm{C}$ & 215 & 303 & \\
\hline $\mathrm{B}_{2} \mathrm{O}_{3}$ & 10 & $\mathrm{~T}_{\mathrm{L}},{ }^{\circ} \mathrm{C}$ & 0.72 & 4 & 1050 \\
\hline $\mathrm{Fe}_{2} \mathrm{O}_{3}$ & 6 & Visc $_{1150}, \mathrm{~Pa} . \mathrm{S}$ & $71.8 \%$ & $62 \%$ & \\
\hline $\mathrm{Li}_{2} \mathrm{O}$ & 6 & Nepheline, $\mathrm{N}_{\mathrm{Si}}$ & & & \\
\hline $\mathrm{Na}_{2} \mathrm{O}$ & 22 & & & & \\
\hline $\mathrm{SiO}_{2}$ & 56 & & & \\
\hline
\end{tabular}

Table 9 Low Viscosity Glass B (with aluminum)

\begin{tabular}{|l|l|l|r|r|r|}
\hline GF/Oxide & wt $\%$ & Property & Value & Minimum & Maximum \\
\hline $\mathrm{Al}_{2} \mathrm{O}_{3}$ & 5 & $\mathrm{~T}_{1 \%}-\mathrm{Sp},{ }^{\circ} \mathrm{C}$ & 359 & 359 & \\
\hline $\mathrm{B}_{2} \mathrm{O}_{3}$ & 10 & $\mathrm{~T}_{\mathrm{L}},{ }^{\circ} \mathrm{C}$ & 1.4 & 4 & 1050 \\
\hline $\mathrm{Fe}_{2} \mathrm{O}_{3}$ & 6 & Visc $_{1150}, \mathrm{~Pa} . \mathrm{S}$ & $67.9 \%$ & $62 \%$ & \\
\hline $\mathrm{Li}_{2} \mathrm{O}$ & 6 & Nepheline, $\mathrm{N}_{\mathrm{Si}}$ & & & \\
\hline $\mathrm{Na}_{2} \mathrm{O}$ & 20 & & & & \\
\hline $\mathrm{SiO}_{2}$ & 53 & & & & \\
\hline
\end{tabular}

Logic for reducing $\mathrm{M}_{\mathrm{Glass.p4}}$ with a low viscosity glass follows.

IF $\mathrm{g}_{\mathrm{Al203.p3}}>0.06$

\section{THEN}

$\begin{array}{ll}\mathrm{VG}_{\mathrm{A} 12 \mathrm{O} 3} & =0.0 \\ \mathrm{VG}_{\mathrm{B} 2 \mathrm{O} 3} & =0.10 \\ \mathrm{VG}_{\mathrm{Fe} 2 \mathrm{O} 3} & =0.06 \\ \mathrm{VG}_{\mathrm{Li} 2 \mathrm{O}} & =0.06 \\ \mathrm{VG}_{\mathrm{Na} 2 \mathrm{O}} & =0.22 \\ \mathrm{VG}_{\mathrm{SiO2}} & =0.56 \\ \eta_{\mathrm{VG}} & =0.72\end{array}$

\section{ELSE}

$\begin{array}{ll}\mathrm{VG}_{\mathrm{ALO} 3} & =0.05 \\ \mathrm{VG}_{\mathrm{B} 2 \mathrm{O} 3} & =0.10 \\ \mathrm{VG}_{\mathrm{Fe} 2 \mathrm{O} 3} & =0.06 \\ \mathrm{VG}_{\mathrm{Li2O}} & =0.06 \\ \mathrm{VG}_{\mathrm{Na} 2 \mathrm{O}} & =0.20 \\ \mathrm{VG}_{\mathrm{SiO} 2} & =0.53 \\ \eta_{\mathrm{VG}} & =1.40\end{array}$

END IF

$\begin{array}{ll}X_{\mathrm{VG}} & =\left(\ln (6)-\ln \left(\eta_{\mathrm{p} 4}\right)\right) /\left(\ln \left(\eta_{\mathrm{VG}}\right)-\ln \left(\eta_{\mathrm{p} 4}\right)\right) \\ \mathrm{M}_{\mathrm{Glass.p5}} & =\mathrm{M}_{\mathrm{Glass} . \mathrm{p3}} /\left(1-\mathrm{X}_{\mathrm{VG}}\right) \\ \mathrm{M}_{\mathrm{VG}} & =\mathrm{M}_{\mathrm{Glass.p5}}-\mathrm{M}_{\mathrm{Glass.p3}}\end{array}$




$$
\begin{aligned}
& \mathrm{GF}_{\mathrm{Al} 203 . \mathrm{ps}}=\mathrm{VG}_{\mathrm{Al} 2 \mathrm{O} 3} * \mathrm{M}_{\mathrm{VG}}+\mathrm{GF}_{\mathrm{Al203} . \mathrm{p} 3} \\
& \mathrm{GF}_{\mathrm{Fe} 2 \mathrm{O3.p5}}=\mathrm{VG}_{\mathrm{Fe} 2 \mathrm{O} 3} * \mathrm{M}_{\mathrm{VG}}+\mathrm{GF}_{\mathrm{Fe} 203 . \mathrm{p} 3} \\
& \mathrm{GF}_{\mathrm{Li} 2 \mathrm{O} \text {. } 5} \quad=\mathrm{VG}_{\mathrm{Li2O}} * \mathrm{M}_{\mathrm{VG}}+\mathrm{MIN}\left[\left(\mathrm{GF}_{\mathrm{L} \text { i2O.p3 }}+\mathrm{M}_{\mathrm{Li} 2 \mathrm{O}}\right) / \mathrm{M}_{\mathrm{Glass} . \mathrm{p} 3}+\Delta_{\mathrm{Li}}, 0.06\right] * \mathrm{M}_{\mathrm{Glass.p.3}}-\mathrm{M}_{\mathrm{Li2O}} \\
& \mathrm{GF}_{\mathrm{B} 203, \mathrm{p} 5} \quad=\mathrm{GF}_{\mathrm{B} 203, \mathrm{p} 3}-\left(\mathrm{GF}_{\mathrm{Li20.p5}}-\mathrm{GF}_{\mathrm{Li20.p3}}\right) \\
& \mathrm{GF}_{\mathrm{Na} 20 . \mathrm{p} 5} \quad=\mathrm{VG}_{\mathrm{Na} 2 \mathrm{O}} * \mathrm{MVG}_{\mathrm{VG}}+\mathrm{GF}_{\mathrm{Na} 2 \mathrm{O} . \mathrm{p} 3} \\
& \mathrm{GF}_{\mathrm{SiO} 2 \mathrm{p} 5}=\mathrm{VG}_{\mathrm{SiO} 2} * \mathrm{M}_{\mathrm{VG}}+\mathrm{GF}_{\mathrm{SiO} 2 \mathrm{p} 3}
\end{aligned}
$$

where: $\mathrm{M}_{\mathrm{VG}}$ is the mass of a high or low viscosity glass added to adjust the viscosity.

$\mathrm{VG}_{i}$ is the mass of glass former to help make the viscosity glass to adjust the viscosity.

The final viscosity of the HLW Glass is given in Equation 34.

Equation 34

$\ln \left(\eta_{\text {Glass }, p 5}\right)=\sum_{i=1}^{19} c_{i} g_{i, p 5}+c_{20} g_{\text {Na2O }} g_{B 2 O 3 . p 5}+c_{21} g_{N a 2 O} g_{A l 203 . p 5}+c_{22} g_{B 2 O 3 . p 5}^{2}+c_{23} g_{C a O . p 5} g_{A l 2 O 3 . p 5}+c_{24} g_{\text {Li2O.p5 }}^{2}$

GlassProp $_{5}=5$

GOTO Product Consistency Test

\section{Low Viscosity Adjustment}

If the viscosity of the PreGlass $\left(\eta_{\mathrm{pg}}\right)$ from Equation 24 is lower than the desired low viscosity $\left(\eta_{\mathrm{L}}\right)$ then the viscosity of the PreGlass needed to be increased to the desired low viscosity $\left(\eta_{L}\right)$. The additive $\mathrm{B}_{2} \mathrm{O}_{3}$ will be exchanged for the additive $\mathrm{SiO}_{2}$, kilogram for kilogram until $\eta_{\mathrm{pg}}$ equals $\eta_{\mathrm{L}}$. The terms that are affected in Equation 34 are given in Equation 35. Exchanging $\mathrm{B}_{2} \mathrm{O}_{3}$ for $\mathrm{SiO}_{2}$ will increase the viscosity because $\mathrm{Si}_{2} \mathrm{O}$ 's coefficients are more positive than $\mathrm{B}_{2} \mathrm{O}_{3}$ 's coefficients. Either $\mathrm{SiO}_{2}$ or $\mathrm{B}_{2} \mathrm{O}_{3}$ can represent the mass change since they are being exchanged kilogram for kilogram but one is the negative of the other as shown in Equation 36. Adding terms for the exchange of some $\mathrm{SiO}_{2}$ for $\mathrm{B}_{2} \mathrm{O}_{3}$ from Equation 37 in Equation 36 gives Equation 38 with just one unknown $\left(\Delta_{\mathrm{Si2O}}\right)$. However, this unknown is not easily solved directly. The value for $\Delta_{\mathrm{Si} \text { O }}$ will be determined numerically by using Newton's Method'.

Adding $\mathrm{SiO}_{2}$ and reducing $\mathrm{B}_{2} \mathrm{O}_{3}$ will increase the $\mathrm{T}_{1 \%}$ value. However, this is not a significant concern because low viscosity glasses generally have low $\mathrm{T}_{1 \%}$ values and can tolerate the exchange of $\mathrm{SiO}_{2}$ and $\mathrm{B}_{2} \mathrm{O}_{3}$ and still be below a $\mathrm{T}_{1 \%}$ value of $950^{\circ} \mathrm{C}$.

Unlike adjustment for high viscosity glasses, a dilution routine for low glasses is not needed for normal operation of the WTP. However, a dilution routine is given in Appendix A. A dilution routine would be needed if glasses made with a low initial $\mathrm{B}_{2} \mathrm{O}_{3}$ concentration (i.e., igf $_{2}$ or $\mathrm{GF}_{\mathrm{B} 203 . \mathrm{pl}}=0.05$ ) were desired.

Equation 35

$$
\ln \left(\eta_{S i B}\right)=c_{2} g_{B 2 O 3, p 3}+c_{14} g_{S i O 2 . p 3}+c_{20} g_{N a 20, p 3} g_{B 2 O 3, p 3}+c_{22} g_{B 2 O 3 . p 3}^{2}
$$

where $c_{i}$ coefficients come from Table 7. 
$\ln \left(\eta_{\Delta S i B}\right)=c_{2}\left(g_{B 2 O 3, p 3}-\Delta_{B 2 O 3}\right)-c_{2} g_{B 2 O 3, p 3}+c_{14}\left(g_{S i O 2, p 3}+\Delta_{S i O 2}\right)-c_{14} g_{S i O 2 . p 3}+c_{20} g_{N a 2 O, p 3}\left(g_{B 2 O 3 . p 3}-\Delta_{B 2 O 3}\right)-c_{20} g_{N a 2 O, p 3} g_{B 2 O 3, p 3}$
$+c_{22}\left(g_{B 2 O 3, p 3}-\Delta_{B 2 O 3}\right)^{2}-c_{22} g_{B 2 O 3 . p 3}^{2}$

Equation 37

$\Delta_{S i 2 O}=\Delta_{B 2 O 3}$

Equation 38

$\ln \left(\eta_{\Delta S B B}\right)=c_{2}\left(g_{B 2 O 3 . p 3}-\Delta_{S i O 2}\right)-c_{2} g_{B 2 O 3 . p 3}+c_{14}\left(g_{S i O 2 . p 3}+\Delta_{S i O 2}\right)-c_{14} g_{S i O 2, p 3}+c_{20} g_{N a 2 O . p 3}\left(g_{B 2 O 3 . p 3}-\Delta_{S i O 2}\right)-c_{20} g_{\text {Na2O.p3 }} g_{B 2 O 3 . p 3}$ $+c_{22}\left(g_{B 2 O 3 . p 3}-\Delta_{S 1 O 2}\right)^{2}-c_{22} g_{B 203 . p 3}^{2}$

or

$\ln \left(\eta_{\Delta S B B}\right)=-c_{2} \Delta_{S O O 2}+c_{14} \Delta_{S i O 2}-c_{20} g_{N a 2 O p_{3}} \Delta_{S i O 2}+c_{22}\left|\left(g_{B 2 O 3 . p 3}-\Delta_{S i O 2}\right)^{2}-g_{B 2 O 3, p 3}^{2}\right|$

\section{Low Viscosity Subroutine}

$\Delta_{0}=0.01$

$\mathrm{DO} n=0$ to 5

$$
\begin{aligned}
& \left.f\left(\Delta_{n}\right)=-c_{2} \Delta_{n}+c_{14} \Delta_{n}-c_{20} g_{\text {Na2O.p3 }} \Delta_{n}+c_{22} \mid\left(g_{B 2 O 3 . p 3}-\Delta_{n}\right)^{2}-g_{B 203 . p 3}^{2}\right\rfloor-\left(\ln \left(\eta_{L}\right)-\ln \left(\eta_{G . p 3}\right)\right) \\
& f^{\prime}\left(\Delta_{n}\right)=-c_{2}+c_{14}-c_{20} g_{N a 2 O}-2 c_{22} g_{B 2 O 3}+2 c_{22} \Delta_{n} \\
& \Delta_{n+1}=\Delta_{n}-\frac{f\left(\Delta_{n}\right)}{f^{\prime}\left(\Delta_{n}\right)}
\end{aligned}
$$

\section{NEXT $n$}

END DO

$$
\begin{aligned}
& \Delta_{\mathrm{SiO2}}=\Delta_{\mathrm{n}+6} \\
& \mathrm{M}_{\mathrm{Glass.} .5}=\mathrm{M}_{\text {Glass.p3 }} \\
& \mathrm{GF}_{\mathrm{Al203.p5}}=\mathrm{GF}_{\mathrm{Al203}, \mathrm{p3}} \\
& \mathrm{GF}_{\mathrm{B} 2 \mathrm{O}, \mathrm{p} 5}=\mathrm{GF}_{\mathrm{B} 2 \mathrm{O} 3 \mathrm{p} 33}-\Delta_{\mathrm{SiO2}} * \mathrm{M}_{\mathrm{Glass}, 3} \\
& \mathrm{GF}_{\mathrm{Fe} 203, \mathrm{p} 5}=\mathrm{GF}_{\mathrm{Fe} 203 . \mathrm{p3}} \\
& \mathrm{GF}_{\mathrm{L} \text {,20,p5 }}=\mathrm{GF}_{\mathrm{L} \text { i20.p3 }} \\
& \mathrm{GF}_{\mathrm{Na} 20, \mathrm{p} 5} \quad=\mathrm{GF}_{\mathrm{Na} 2 \mathrm{O} . \mathrm{p3}} \\
& \mathrm{GF}_{\mathrm{SiO} 2 \mathrm{ps}}=\mathrm{GF}_{\mathrm{SiO} 2 \mathrm{p} 3}+\Delta_{\mathrm{SiO2}} * \mathrm{M}_{\mathrm{Glass} .3} \\
& \ln (\eta \text { Glass.p5 })=\ln (4) \\
& \text { GlassProp }_{6}=6
\end{aligned}
$$

GO TO Product Consistency Test 


\section{Product Consistency Test}

There are three Product Consistency Tests (PCTs) which the HLW glass must pass. The PCT is a leaching procedure that tests for leached boron, sodium, and lithium in the leachate from the tests. These tests are new to the HLW Glass Shell routine. Most batches of HLW glass as formulated by the HLW Glass Shell routine will pass the three PCT tests. However, a few batches may not. The following routines check for these failed batches and then dilute glass $M_{\text {glass.ps }}$ with a good glass low in PCT values until $\mathrm{M}_{\text {glass.p5 }}$ passes and becomes glass $\mathrm{M}_{\text {glass.p6 }}$ for Liquidus Temperature, $T_{L}$ Model routine.

The PCT routine starts with calculating the PCT values for boron, sodium, and lithium per Equation 39 and Equation 40 using glass $M_{\text {glass.ps }}$ values.

Equation 39

$$
\begin{aligned}
& \ln (P C T)=\sum_{i=1}^{23} d_{i} g_{i, p 5}+d_{24} g_{A l 2 O 3 . p 5}^{2}+d_{25} g_{A 1203, p 5} g_{T h O 2, p 5}+d_{26} g_{A i 2 O 3, p 5}^{3}+c_{27} g_{B 2 O 3, p 5}^{2}+c_{28} g_{M n O . p 5}^{2} \\
& +c_{29} g_{B 203, p 5} g_{N a 20, p 5}
\end{aligned}
$$

Equation 40

$$
P C T=\exp [\ln (P C T)]
$$

where:

$$
\ln (\mathrm{PCT}) \text { is the natural } \log \text { of the PCT value. The PCT values are in } \mathrm{g} / \mathrm{m}^{2} \text { of leached }
$$
boron, sodium, or lithium. The subscript G.p5 means the PCT of the PreGlass $\left(\mathrm{M}_{\mathrm{Glass} . \mathrm{p}}\right)$.

$i$ is the $i^{\text {th }}$ component in Table 1 .

$\mathrm{g}_{i}$ is the mass fraction of the component in the PreGlass $\left(\mathrm{M}_{\mathrm{Glass} . \mathrm{p} 5}\right)$, i.e., $\mathrm{g}_{1}=\left(\mathrm{GF}_{\mathrm{Al203.p5}}+\right.$ $\left.\mathrm{M}_{\mathrm{Aj} 2 \mathrm{O} 3}\right) / \mathrm{M}_{\mathrm{Glass} . \mathrm{p} 5}$

$\mathrm{d}_{\mathrm{i}}$ is the coefficient of the $i^{\text {th }}$ component.

$\mathrm{M}_{\text {othersPCT }}=\mathrm{M}_{\text {others }}-\mathrm{M}_{\mathrm{BaO}}-\mathrm{M}_{\mathrm{BizO} 3}-\mathrm{M}_{\mathrm{CaO}}-\mathrm{M}_{\mathrm{CdO}}-\mathrm{M}_{\mathrm{F}}-\mathrm{M}_{\mathrm{K} 2 \mathrm{O}}-\mathrm{M}_{\mathrm{MgO}}-\mathrm{M}_{\mathrm{MnO}}$

$$
-\mathrm{M}_{\mathrm{Nd} 2 \mathrm{O} 3}-\mathrm{M}_{\mathrm{P} 2 \mathrm{O} 5}-\mathrm{M}_{\mathrm{SO} 3}-\mathrm{M}_{\mathrm{SrO}}-\mathrm{M}_{\mathrm{ThO} 2}-\mathrm{M}_{\mathrm{TiO} 2}-\mathrm{M}_{\mathrm{UO} 3}-\mathrm{M}_{\mathrm{ZnO}}-\mathrm{M}_{\mathrm{ZrO} 2}
$$


Table 10 Product Consistency Test (PCT) Coentients

\begin{tabular}{|c|c|c|c|c|}
\hline$(i)$ & Term & $\ln [$ PCT-B $], \mathbf{d}_{i}$ & $\ln [\mathrm{PCT}-\mathrm{Na}], \mathrm{d}_{i}$ & $\ln \left[\right.$ PCT-Li], $d_{i}$ \\
\hline 1 & $\mathrm{Al}_{2} \mathrm{O}_{3}$ & -88.2711 & -69.768 & -71.80358 \\
\hline 2 & $\mathrm{~B}_{2} \mathrm{O}_{3}$ & 13.01511 & -13.224 & -15.88115 \\
\hline 3 & $\mathrm{BaO}$ & 5.657878 & 0 & 0 \\
\hline 4 & $\mathrm{CaO}$ & -3.38958 & 2.60598 & 0.4612474 \\
\hline 5 & $\mathrm{CdO}$ & 12.66478 & 0 & 0 \\
\hline 6 & $\mathrm{~F}$ & 28.72152 & 25.9327 & 29.676446 \\
\hline 7 & $\mathrm{Fe}_{2} \mathrm{O}_{3}$ & -1.97003 & -1.6767 & -0.588641 \\
\hline 8 & $\mathrm{~K}_{2} \mathrm{O}$ & 10.91193 & 11.9701 & 10.211474 \\
\hline 9 & $\mathrm{Li}_{2} \mathrm{O}$ & 26.08455 & 22.5717 & 24.236504 \\
\hline 10 & $\mathrm{MgO}$ & 10.32971 & 9.44187 & 6.8614839 \\
\hline 11 & $\mathrm{MnO}$ & -11.942 & 1.43131 & 0 \\
\hline 12 & $\mathrm{Na}_{2} \mathrm{O}$ & 15.66601 & 16.9404 & 5.8154351 \\
\hline 13 & $\mathrm{Nd}_{2} \mathrm{O}_{3}$ & -6.94385 & -2.8519 & 0 \\
\hline 14 & $\mathrm{P}_{2} \mathrm{O}_{5}$ & -3.93437 & -2.3946 & 0 \\
\hline 15 & $\mathrm{SiO}_{2}$ & -3.27355 & -2.0965 & -0.63572 \\
\hline 16 & $\mathrm{SO}_{3}$ & 26.09692 & 22.3441 & 42.822925 \\
\hline 17 & $\mathrm{SrO}$ & -1.69883 & 2.04919 & 0 \\
\hline 18 & $\mathrm{ThO}_{2}$ & -14.2188 & -13.455 & -14.11654 \\
\hline 19 & $\mathrm{TiO}_{2}$ & -11.0803 & -11.347 & -15.98701 \\
\hline 20 & $\mathrm{UO}_{3}$ & 2.505961 & 1.49105 & 0 \\
\hline 21 & $\mathrm{ZnO}$ & 0.548053 & -0.9934 & 0 \\
\hline 22 & $\mathrm{ZrO}_{2}$ & -6.44919 & -5.2997 & -4.149619 \\
\hline 23 & Others TCP & -0.41991 & 2.73564 & 2.1636268 \\
\hline 24 & $\left(\mathrm{Al}_{2} \mathrm{O}_{3}\right)^{2}$ & 705.3445 & 557.465 & 586.1739 \\
\hline 25 & $\mathrm{Al}_{2} \mathrm{O}_{3} \times \mathrm{ThO}_{2}$ & 193.7515 & 201.149 & 236.62771 \\
\hline 26 & $\left(\mathrm{Al}_{2} \mathrm{O}_{3}\right)^{3}$ & -1974.56 & -1590.6 & -1640.968 \\
\hline 27 & $\left(\mathrm{~B}_{2} \mathrm{O}_{3}\right)^{2}$ & 0 & 96.3525 & 88.683572 \\
\hline 28 & $(\mathrm{MnO})^{2}$ & 286.0626 & 0 & 0 \\
\hline 29 & $\mathrm{~B}_{2} \mathrm{O}_{3} \times \mathrm{Na}_{2} \mathrm{O}$ & 0 & 0 & 74.407878 \\
\hline
\end{tabular}

The desired PCT values for boron, sodium, and lithium is $4.0 \mathrm{~g} / \mathrm{m}^{2}$ or less. Glass $M_{\text {glass. }}$ will be adjusted for the highest value of the three over $4.0 \mathrm{~g} / \mathrm{m}^{2}$. If none are over $4.0 \mathrm{~g} / \mathrm{m}^{2}$, no adjustment will be made and the new glass $\mathbf{M}_{\text {glass. } 6}$ equals $\mathbf{M}_{\text {glass.5. }}$.

Table 11 PCT Dilution Glass

\begin{tabular}{|c|c|c|c|c|c|}
\hline GF/Oxide & $w t \%$ & Property & Value & Minimum & Maximum \\
\hline $\mathrm{Al}_{2} \mathrm{O}_{3}$ & 8 & $\mathrm{~T}_{1 \%}-\mathrm{Sp},{ }^{\circ} \mathrm{C}$ & 582 & & 950 \\
\hline $\mathrm{B}_{2} \mathrm{O}_{3}$ & 10 & $\mathrm{~T}_{\mathrm{L}},{ }^{\circ} \mathrm{C}$ & 584 & & 1050 \\
\hline $\mathrm{Fe}_{2} \mathrm{O}_{3}$ & 9 & $\operatorname{Visc}_{1150}, \mathrm{~Pa} . \mathrm{s}$ & 4.82 & 4 & 6 \\
\hline $\mathrm{Li}_{2} \mathrm{O}$ & 2 & Nepheline, $\mathrm{N}_{S i}$ & $62.0 \%$ & $62 \%$ & \\
\hline $\mathrm{Na}_{2} \mathrm{O}$ & 22 & $\mathrm{PCT}-\mathrm{B}, \mathrm{g} / \mathrm{m}^{2}$ & 0.93 & & 4 \\
\hline $\mathrm{SiO}_{2}$ & 49 & $\mathrm{PCT}-\mathrm{Na}, \mathrm{g} / \mathrm{m}^{2}$ & 0.61 & & 4 \\
\hline & & PCT-Li, g/m $\mathrm{m}^{2}$ & 0.83 & & 4 \\
\hline
\end{tabular}

The PCT Dilution Glass is shown in Table 11. Notice that this glass has different PCT values for boron, sodium, and lithium. So the amount of dilution glass needed will vary depending on the PCT value. The following logic determines how much PCT Dilution Glass to use. 
PCT.p5 = MAX (PCT-B, PCT-Na, PCT-Li)

where: PCT-B, PCT-Na, and PCT-Li are the values per Equation 40.

IF PCT.p5 > 4.0 THEN \{Glass need PCT glass dilution\}

(c IF THEN)

IF (PCT-B > MAX (PCT-Na, PCT-Li) THEN $\quad$ PCT-B applies

(d IF THEN)

DilutePCT $=0.93$

GlassProp $_{8}=8$

GOTO PCT Dilution Glass Subroutine

ELSE

(d ELSE)

IF (PCT-Na > PCT-LI) THEN \{PCT-Na applies\}

(e IF THEN)

DilutePCT $=0.61$

GlassProp $_{9}=9$

GOTO PCT Dilution Subroutine

ELSE \{PCT-Li applies\}

(e ELSE)

DilutePCT $=0.83$

GlassProp $_{10}=10$

END IF

(e END IF)

END IF

(d END IF)

PCT Dilution Glass Subroutine

$$
\begin{aligned}
& \mathrm{MpctG}=\mathrm{M}_{\mathrm{Glass} . \mathrm{p5}} *(\ln (\mathrm{PCT} . \mathrm{p} 5)-\ln (4)) /(\ln (4)-\ln (\text { DilutionPCT })) \\
& \mathrm{GF}_{\mathrm{Al} 203, \mathrm{p} 6}=0.08 * \mathrm{MpctG}+\mathrm{GF}_{\mathrm{Al203.p5}} \\
& \mathrm{GF}_{\mathrm{B} 203 . \mathrm{p} 6}=0.10 * \mathrm{MpctG}+\mathrm{GF}_{\mathrm{B2} 23 . \mathrm{ps}} \\
& \mathrm{GF}_{\mathrm{Fe} 203 . \mathrm{p} 6}=0.09 * \mathrm{MpctG}+\mathrm{GF}_{\mathrm{Fe} 203 . \mathrm{p} 5} \\
& \mathrm{GF}_{\mathrm{Li20.p6}}=0.02 * \mathrm{MpctG}+\mathrm{GF}_{\mathrm{Li20.p5}} \\
& \mathrm{GF}_{\mathrm{Na} 20 . \mathrm{p} 6 \quad}=0.22 * \mathrm{MpctG}+\mathrm{GF}_{\mathrm{Na} 20 . \mathrm{p} 5} \\
& \mathrm{GF}_{\mathrm{SiO2,p6}} \quad=0.49 * \mathrm{MpctG}+\mathrm{GF}_{\mathrm{SiO2}, \mathrm{ps}} \\
& \mathrm{M}_{\mathrm{Glass} . \mathrm{p} 6} \quad=\mathrm{MpctG}+\mathrm{M}_{\mathrm{Glass} . \mathrm{p} 5} \\
& \text { PCT.p6 }=4.0
\end{aligned}
$$




\section{GOTO Liquid Temperature Model}

Note: Since the PCT model contains some square terms and even a cubic terms (see Equation 39) the dilution routines tends to overshoot a small amount. This means the actual PCT for glass $\mathrm{M}_{\text {Glass.p6 }}$ will actually be a little less than 4.0 .

ELSE \{Glass does not need PCT Glass Dilution\}

(c ELSE)

$$
\begin{aligned}
& \mathrm{M}_{\text {Glass.p6 }} \quad=\mathrm{M}_{\text {Glass.p5 }} \\
& \mathrm{GF}_{\mathrm{Al} 203 . \mathrm{p} 6}=\mathrm{GF}_{\mathrm{Al} 203 . \mathrm{p} 5} \\
& \mathrm{GF}_{\mathrm{B} 203, \mathrm{p} 6}=\mathrm{GF}_{\mathrm{B} 203, \mathrm{p5}} \\
& \mathrm{GF}_{\mathrm{Fe} 203 . \mathrm{p} 6}=\mathrm{GF}_{\mathrm{Fe} 203 . \mathrm{p} 5} \\
& \mathrm{GF}_{\mathrm{L} \text { 220.p6 }}=\mathrm{GF}_{\mathrm{Li20.p5}} \\
& \mathrm{GF}_{\mathrm{Na} 20 . p 6}=\mathrm{GF}_{\mathrm{Na} 20 . p 5} \\
& \mathrm{GF}_{\mathrm{Si} 02, \mathrm{p} 6}=\mathrm{GF}_{\mathrm{Si} 2, \mathrm{pj}} \\
& \text { PCT.p6 = PCT.p5 }
\end{aligned}
$$

END

\{Ends PCT Dilution Subroutine\} ～(c END)

\section{Liquidus Temperature, $\mathbf{T}_{\mathrm{L}}$, Model}

The formation of zircon can happen in HLW glasses and can affect glass chemistry. The model for zircon liquidus temperature follows the same format as the $T_{1 \%}$.. $T_{L}$ for PreGlass $\left(M_{G l a s s .6}\right)$ should be within limits or close because of all the adjustments made to the glass thus far. However, if adjustment is necessary it is due to high concentrations of $\mathrm{Al}_{2} \mathrm{O}_{3}, \mathrm{SrO}_{2}, \mathrm{~B}_{2} \mathrm{O}_{3}$, and $\mathrm{ZrO}_{2}$. Consequently, viscosity will be at the high end and PCT, etc. will be at the low end. Therefore, swapping $\mathrm{Na}_{2} \mathrm{O}$ for $\mathrm{B}_{2} \mathrm{O}_{3}$ will be used. Swapping these components should adjust the glass properties in the positive directions without impact on waste loading. Go to the $T_{L}$ Adjustment Glass Subroutine below to make the final adjustment to the HLW glass.

Equation 4

$$
T_{\text {L.p } 6}=\sum_{i=1}^{7} \mathrm{~d}_{\mathrm{i}} \frac{M_{i}+G F_{i}}{M_{\text {Glass. } p 6}}+d_{8} \frac{M_{\text {othersTL }}}{M_{\text {Glass. } 66}}
$$

where: $\quad T_{\mathrm{L} . \mathrm{p} 6}$ is the Liquidus Temperature for PreGlass $\left(\mathrm{M}_{\mathrm{Glass} . \mathrm{p} 6}\right)$.

$i$ is the $i^{\text {th }}$ component in Table 12 .

$\mathrm{M}_{\mathrm{i}}$ is the Mass of $i$-th waste component listed in Table 12. There are 7 components plus Others.

$\mathrm{GF}_{\mathrm{i}}$ is the Mass of the $i$-th glass former component $\left(\mathrm{GF}_{\mathrm{ip} 6}\right)$ matching the waste component listed in Table 12.

$\mathrm{d}_{i}$ is the component coefficient for $i$-th component in Table 12 . 
Table $12 \quad T_{L},{ }^{\circ} \mathrm{C} / \mathrm{w} \%$ Model Coefficients

\begin{tabular}{|c|c|c|}
\hline$(\boldsymbol{i})$ & $\mathbf{g}_{\boldsymbol{i}}$ & Coefficient $\left(\mathbf{b}_{\boldsymbol{i}}\right)$ \\
\hline 1 & $\mathrm{Al2O} 3$ & 3193.3628 \\
\hline 2 & $\mathrm{~B} 2 \mathrm{O} 3$ & 651.39721 \\
\hline 3 & $\mathrm{Ln} 2 \mathrm{O} 3$ & 2156.4074 \\
\hline 4 & $\mathrm{Li2O}$ & -1904.417 \\
\hline 5 & $\mathrm{Na2O}$ & -1947.711 \\
\hline 6 & SrO & 13011.909 \\
\hline 7 & ZrO2 & 3747.4241 \\
\hline 8 & Others & 1259.2233 \\
\hline
\end{tabular}

Equation 42

$\mathrm{M}_{\mathrm{Ln} 2 \mathrm{O} 3}=\mathrm{M}_{\mathrm{Y} 2 \mathrm{O} 3}+\mathrm{M}_{\mathrm{La} 2 \mathrm{O} 3}+\mathrm{M}_{\mathrm{Ce} 2 \mathrm{O} 3}+\mathrm{M}_{\mathrm{PR2O3}}+\mathrm{M}_{\mathrm{Nd2O3}}+\mathrm{M}_{\mathrm{Sm} 2 \mathrm{O} 3}+\mathrm{M}_{\mathrm{Gd} 2 \mathrm{O} 3}$ (for use with $i=3$ in Table 12.)

Equation 43

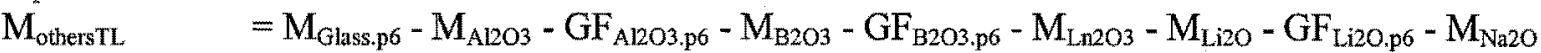
$-\mathrm{GF}_{\mathrm{Na} 2 \mathrm{O} . \mathrm{p} 6}-\mathrm{M}_{\mathrm{SrO}}-\mathrm{M}_{\mathrm{ZrO2}}$

Equation 44 shows how much $\mathrm{B}_{2} \mathrm{O}_{3}$ and $\mathrm{Na}_{2} \mathrm{O}$ need to be changed to bring $\mathrm{T}_{\mathrm{L}}$ down to the liquidus temperature limit, $\mathrm{T}_{\mathrm{L} \text {-limit. }}$ Since $\mathrm{B}_{2} \mathrm{O}_{3}$ and $\mathrm{Na}_{2} \mathrm{O}$ will be swapped $\mathrm{kg}$ for $\mathrm{kg}$. Equation 44 can be solved for $\Delta \mathrm{Na}_{2} \mathrm{O}$ as shown in Equation 45 .

Equation 4

$\mathrm{T}_{\text {L-limit }}-\mathrm{T}_{\mathrm{L}, \mathrm{p} 6}=\mathrm{d}_{2} \Delta \mathrm{B}_{2} \mathrm{O}_{3}+\mathrm{d}_{5} \Delta \mathrm{Na}_{2} \mathrm{O}$

Equation 45

$\triangle \mathrm{Na}_{2} \mathrm{O}$

$$
=\left(T_{\text {L-limit }}-T_{L . p 6}\right) /\left(\mathrm{d}_{5}-\mathrm{d}_{2}\right)
$$

\section{TL Adjustment Glass Subroutine}

IF $\mathrm{T}_{\mathrm{L} p \mathrm{p} 6}<=1050$ THEN

$$
\begin{array}{ll}
\mathrm{M}_{\text {Glass.p7 }} & =\mathrm{M}_{\mathrm{Glass} . \mathrm{p} 6} \\
\mathrm{GF}_{\mathrm{AL203.p7}} & =\mathrm{GF}_{\mathrm{AL203.p6}} \\
\mathrm{GF}_{\mathrm{B} 203 . \mathrm{p} 7} & =\mathrm{GF}_{\mathrm{B} 203 . \mathrm{p} 6} \\
\mathrm{GF}_{\mathrm{Fe} 203 . \mathrm{p} 7} & =\mathrm{GF}_{\mathrm{Fe} 203 . \mathrm{p} 6} \\
\mathrm{GF}_{\mathrm{L} \text { L20.p7 }} & =\mathrm{GF}_{\mathrm{Li20.p6}} \\
\mathrm{GF}_{\mathrm{Na20.p7}} & =\mathrm{GF}_{\mathrm{Na20.p6}} \\
\mathrm{GF}_{\mathrm{SiO2.p7}} & =\mathrm{GF}_{\mathrm{S} 102 . p 6} \\
\mathrm{P}_{\mathrm{T} . \mathrm{p} 7} & =\mathrm{P}_{\mathrm{T}} . \mathrm{p} 6
\end{array}
$$

GOTO Print Subroutine 


\section{ELSE}

$$
\begin{aligned}
& \left.\Delta \mathrm{Na}_{2} \mathrm{O}=\mathrm{M}_{\mathrm{Glass} . \mathrm{p} 6} *\left(1050-\mathrm{T}_{\mathrm{L} . \mathrm{p} 6}\right) /\left(\mathrm{d}_{5}-\mathrm{d}_{2}\right)\right) \\
& \mathrm{M}_{\text {Glass.p7 }} \quad=\mathrm{M}_{\mathrm{Glass} . \mathrm{p} 6} \\
& \mathrm{GF}_{\mathrm{A} 1203 . \mathrm{p} 7}=\mathrm{GF}_{\mathrm{Al203} . \mathrm{p} 6} \\
& \mathrm{GF}_{\mathrm{B} 203 . \mathrm{p} 7}=\mathrm{GF}_{\mathrm{B} 203 . \mathrm{p} 6}-\Delta \mathrm{Na}_{2} \mathrm{O} \\
& \mathrm{GF}_{\mathrm{Fe} 203 . \mathrm{p} 7}=\mathrm{GF}_{\mathrm{Fe} 203 . \mathrm{p} 6} \\
& \mathrm{GF}_{\text {Li20.p } 7}=\mathrm{GF}_{\text {Li20.p6 }} \\
& \mathrm{GF}_{\mathrm{Na} 2 \mathrm{O} . \mathrm{p} 7}=\mathrm{GF}_{\mathrm{Na} 2 \mathrm{O} . \mathrm{p} 6}+\Delta \mathrm{Na}_{2} \mathrm{O} \\
& \mathrm{GF}_{\mathrm{SiO2.p7}}=\mathrm{GF}_{\mathrm{SiO} . \mathrm{p} 6} \\
& \mathrm{~T}_{\mathrm{L}} \cdot \mathrm{p} 7 \quad=1050 \\
& \text { GlassProp }_{11}=11
\end{aligned}
$$

END

\{Ends Liquidus Temperature Subroutine\}

\section{Print Subroutine}

With the Dynamic Model values can be lost if they are not specifically printed to a file. This data is important in verifying logic and producing simulated operational data for charts and reports. The following values shall be printed to a printout file.

$M_{\text {waste }}, M_{\text {others }}, M_{\text {others } 1 \%}, M_{\text {othersn }}, M_{\text {othersPCT }}, M_{i}$ for each of the waste oxides,

$\mathrm{M}_{\text {Glass.p1 }}, \mathrm{M}_{\mathrm{Glass} . \mathrm{p} 2}, \mathrm{M}_{\mathrm{Glass} . \mathrm{p} 3}, \mathrm{M}_{\mathrm{Glass} . \mathrm{p} 4}, \mathrm{M}_{\mathrm{Glass} . \mathrm{p5}}, \mathrm{M}_{\mathrm{Glass} . \mathrm{p6}}, \mathrm{BC}_{\mathrm{MAX}}, \mathrm{BC}_{\mathrm{Flag}}, \mathrm{BC}_{i}$ series

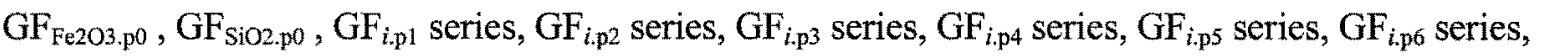
$\mathrm{GF}_{i . \mathrm{p} 7}$ series

LimitProp , GlassProp Series $N_{\text {nap }}, T_{1 \% . p^{2}}, T_{1 \%, p^{3}}$, MaxAlFe $, M_{D G}, \ln \left(\eta_{G . p 3}\right), \ln \left(\eta_{G . p 4}\right), \ln \left(\eta_{G . p 5}\right)$, $\mathrm{g}_{\mathrm{Li20}, \mathrm{p3}}, \Delta_{\mathrm{Li2O}}, \Delta_{\mathrm{Li}}, \Delta_{\mathrm{Li} 20 \mathrm{X}}, \mathrm{X}_{\mathrm{VG}}, \Delta_{\mathrm{Si}}, \Delta_{\mathrm{SiO} 2}$, PCT.p5, PCT.p6, MpctG, $\mathrm{P}_{\mathrm{T}}$.p6, $\mathrm{P}_{\mathrm{T}} . \mathrm{p} 7$

\section{GlassProp Number}

A code, called GlassProp, was place in the HLW Glass Shell routine to quickly summary what glass properties impacted the formulation of the batch. The limiting glass (LimitProp) property for the glass is determined by Equation 46. What the values mean is given in Table 13.

Equation 46

LimitProp $=$ MAX $\left(\right.$ GlassProp $_{0} \ldots$ GlassProp $\left._{11}\right)$ 
Table 13 Description of Digits in the GlassProp

\begin{tabular}{|c|l|}
\hline $\boldsymbol{i}$ & \multicolumn{1}{|c|}{ Property Affecting Glass and Adjustment Made } \\
\hline 0 & None. (Applies only if no other GlassProp $i$ value. Glass limited by $\mathrm{BC}_{i}$ value.) \\
\hline 1 & Low Nepheline Value - $\mathrm{SiO}_{2}$ added \\
\hline 2 & High $\mathrm{T}_{1 \%}$ - Diluted with $\mathrm{T}_{1 \%}$ glass without $\mathrm{Al}_{2} \mathrm{O}_{3}$ and $\mathrm{Fe}_{2} \mathrm{O}_{3}$ \\
\hline 3 & High $\mathrm{T}_{1 \%}$ - Diluted with low $\mathrm{T}_{1 \%}$ glass containing $\mathrm{Al}_{2} \mathrm{O}_{3}$ and $\mathrm{Fe}_{2} \mathrm{O}_{3}$ \\
\hline 4 & High Viscosity $-\mathrm{Li}_{2} \mathrm{O}$ swapped for $\mathrm{B}_{2} \mathrm{O}_{3}$ \\
\hline 5 & High Viscosity $-\mathrm{Li}_{2} \mathrm{O}$ swapped for $\mathrm{B}_{2} \mathrm{O}_{3}$ plus low-viscosity dilution glass used \\
\hline 6 & Low Viscosity $-\mathrm{SiO}_{2}$ swapped for $\mathrm{B}_{2} \mathrm{O}_{3}$ \\
\hline 7 & Low Viscosity $-\mathrm{SiO}_{2}$ swapped for $\mathrm{B}_{2} \mathrm{O}_{3}$ plus viscous dilution glass used \\
\hline 8 & High PCT-B - Dilution glass with low PCT value used \\
\hline 9 & High PCT-B - Dilution glass with low PCT value used \\
\hline 10 & High PCT-B - Dilution glass with low PCT value used \\
\hline 11 & High Liquidus Temperature, $\mathrm{T}_{\mathrm{L}}$, swapped $\mathrm{Na}_{2} \mathrm{O}$ for $\mathrm{B}_{2} \mathrm{O}_{3}$ \\
\hline
\end{tabular}

\section{Other Glass Property Models}

Document PNNL 18501, Glass Property Data and Models for Estimating High-Level Waste Glass

Volume, documents some other glass property models, namely Electrical Conductivity $(\varepsilon)$ and TCLP.

These glass models would have little or no impact on calculating the volume of HLW glass. Justification is given in PNNL 18501. However, the following is a short summary:

Electrical Conductivity - Studies show that if the glass viscosity is good, electrical conductivity of the glass is also good. Consequently, electrical conductivity is not needed for estimating glass volume.

$\square$ TCLP - Historically the glasses contain so little Cadmium (the major TCLP concern) that failure of glass due to high cadmium TCLP values is rare. Consequently, it has little or no impact on estimating WTP mission glass volumes.

\section{References}

PNNL-18501, Glass Property Data and Models for Estimating High-Level Glass Volume, J.D. Vienna, et. al., October 2009.

24590-101-TSA-W000-0009-02-00001, Final Report: Baseline HLW Glass Formulations for Bismuth Phosphate Wastes.

CCN 184900, Non-Spinel Phase Rule - Supersedes CCN 170601, John Vienna, September 16, 2008.

24590-HLW-RPT-RT-05-001, Rev. 0, Preliminary IHLW Formulation Algorithm Description, J.D. Vienna and D. Kim. 


\section{Appendix A}

\section{Low Viscosity Glass Dilution}

Validation of the HLW Shell showed that this Low Viscosity Dilution Subroutine will not be called because the initial boron glass formers $\left(\mathrm{igf}_{2}, \mathrm{GF}_{\mathrm{B} 2 \mathrm{O} \text {.p1 } 1}\right)$ is set at 0.20 there is adequate adjustment with just swapping $\mathrm{SiO}_{2}$ for $\mathrm{B}_{2} \mathrm{O}_{3}$. However, if a low igf $_{2}$ were chosen, a dilution subroutine may be necessary. Preliminary investigation indicates that $\operatorname{igf}_{2}$ would have to be 0.05 for this to happen. This is too for normal use. However, a dilution routine is given here if ever needed. A dilution routine is needed:

IF $\mathrm{g}_{\mathrm{B} 2 \mathrm{O} . \mathrm{p3} 3}-\Delta_{\mathrm{SiO} 2}<0.04$ (i.e., $4 \mathrm{wt} \% \mathrm{~B}_{2} \mathrm{O}_{3}$ in glass) THEN

GOTO Low Viscosity Dilution Subroutine

\section{ELSE}

GO TO Product Consistency Test \{Dilution is not necessary\}

\section{Low Viscosity Dilution Subroutine}

No more $\mathrm{B}_{2} \mathrm{O}_{3}$ can be removed and no more $\mathrm{SiO}_{2}$ can be added to the $\mathrm{M}_{\mathrm{Glass} . \mathrm{p} 3}$ glass. Therefore, this glass needs to be diluted with another glass to further increase the viscosity. Before doing this, however, the viscosity of the new $\mathrm{M}_{\text {Glass.p3 }}$ with the minimum $\mathrm{B}_{2} \mathrm{O}_{3}$ or maximum $\mathrm{SiO}_{2}$ (which is $\mathrm{M}_{\text {Glass.p4 }}$ ) needs to be calculated. Equation 38 can be used to calculate $\mathrm{M}_{\mathrm{Glass} . \mathrm{p} 4}$. Equation 47 shows how much extra silica to use in Equation 38. Equation 38 is rewritten into a more useable equation as shown in Equation 48.

Equation 47

$\Delta_{\text {SiO2X }}=g_{\text {B2O3.p3 }}-0.04$

Equation 48

$\ln \left(\eta_{\text {Glass. } p 4}\right)=\ln \left(\eta_{\text {Glass. } p 3}\right)+\left[\begin{array}{l}c_{2}\left(g_{\text {B2O3.p3 }}-\Delta_{\text {SiO } 2 X}\right)+c_{14}\left(g_{\text {SiO2. } p 3}+\Delta_{\text {SiO } 2 X}\right)+c_{20} g_{\text {Na2O.p3 }}\left(g_{\text {B2O3. } p 3}-\Delta_{\text {SiO } 2 X}\right) \\ +c_{22}\left(g_{\text {B2O3. } p 3}-\Delta_{\text {SiO } X X}\right)^{2}\end{array}\right]$

The viscosity of $\mathrm{M}_{\text {Glass.p4 }}$ glass using the high viscosity glass is shown in Table 14. Logic for doing this follows. Since the viscosity model is nonlinear, this routine is not exact, but should provide a close estimate. It provides glass quantities sufficient for estimating WTP mission values.

Table 14 High Viscosity Glass

\begin{tabular}{|c|c|c|c|c|c|}
\hline GF/Oxide & wt\% & Property & Value & Minimum & Maximum \\
\hline $\mathrm{Al}_{2} \mathrm{O}_{3}$ & 4 & $\mathrm{~T}_{1 \%}-\mathrm{Sp},{ }^{\circ} \mathrm{C}$ & 469 & & 950 \\
\hline $\mathrm{B}_{2} \mathrm{O}_{3}$ & 4 & $\mathrm{~T}_{\mathrm{L}},{ }^{\circ} \mathrm{C}$ & 469 & & 1050 \\
\hline $\mathrm{Fe}_{2} \mathrm{O}_{3}$ & 3 & Visc $_{1150}$, Pa.s & 656 & 4 & 6 \\
\hline $\mathrm{Li}_{2} \mathrm{O}$ & 0 & Nepheline, $\mathrm{N}_{\mathrm{Si}}$ & $84.9 \%$ & $62 \%$ & \\
\hline $\mathrm{Na}_{2} \mathrm{O}$ & 10 & & & & \\
\hline $\mathrm{SiO}_{2}$ & 79 & & & & \\
\hline
\end{tabular}




$$
\begin{aligned}
& \mathrm{VG}_{\mathrm{A} 1203} \quad=0.04 \\
& \mathrm{VG}_{\mathrm{B} 2 \mathrm{O3}} \quad=0.04 \\
& \mathrm{VG}_{\mathrm{Fe} 2 \mathrm{O} 3} \quad=0.03 \\
& \mathrm{VG}_{\mathrm{Li2O}} \quad=0.0 \\
& \mathrm{VG}_{\mathrm{Na} 2 \mathrm{O}} \quad=0.10 \\
& \mathrm{VG}_{\mathrm{SiO} 2} \quad=0.53 \\
& \eta_{\mathrm{VG}}=656 \\
& \mathrm{X}_{\mathrm{VG}} \quad=\left(\ln (4)-\ln \left(\eta_{\text {Glass. } \mathrm{p} 4}\right)\right) /\left(\ln \left(\eta_{\mathrm{VG}}\right)-\ln \left(\eta_{\text {Glass. } \mathrm{p} 4}\right)\right) \\
& \mathrm{M}_{\mathrm{Glass} . \mathrm{ps}} \quad=\mathrm{M}_{\mathrm{Glass} . \mathrm{p4}} / \mathrm{X}_{\mathrm{VG}} \\
& \mathrm{M}_{\mathrm{VG}} \quad=\mathrm{M}_{\mathrm{Glass} . \mathrm{p5}}-\mathrm{M}_{\mathrm{Glass} . \mathrm{p4}} \\
& \mathrm{GF}_{\mathrm{A} 203 \mathrm{p5}}=\mathrm{VG}_{\mathrm{Al} 203} * \mathrm{M}_{\mathrm{VG}}+\mathrm{GF}_{\mathrm{Al} 2 \mathrm{O} 3 \mathrm{p} 3} \\
& \mathrm{GF}_{\mathrm{B} 203 . \mathrm{ps}}=\mathrm{VG}_{\mathrm{B} 2 \mathrm{O3}} * \mathrm{M}_{\mathrm{VG}}+\mathrm{GF}_{\mathrm{B} 203 . \mathrm{p} 3}-\Delta_{\mathrm{SiO2}} * \mathrm{M}_{\mathrm{Glass} .3} \\
& \mathrm{GF}_{\mathrm{Fe} 203 . \mathrm{p} 5}=\mathrm{VG}_{\mathrm{Fe} 203} * \mathrm{M}_{\mathrm{VG}}+\mathrm{GF}_{\mathrm{Fe} 2 \mathrm{O} 3 \mathrm{p3}} \\
& \mathrm{GF}_{\mathrm{Li2O} . \mathrm{p} 5} \quad=\mathrm{VG}_{\mathrm{Li2O}} * \mathrm{M}_{\mathrm{VG}}+\mathrm{GF}_{\mathrm{Li2O}, \mathrm{p} 3} \\
& \mathrm{GF}_{\mathrm{Na} 20 . \mathrm{p} 5}=\mathrm{VG}_{\mathrm{Na} 20} * \mathrm{M}_{\mathrm{VG}}+\mathrm{GF}_{\mathrm{Na} 20 . \mathrm{p} 3} \\
& \mathrm{GF}_{\mathrm{SiO2} \text {. } 55}=\mathrm{VG}_{\mathrm{SiO2} 2} * \mathrm{M}_{\mathrm{VG}}+\mathrm{GF}_{\mathrm{SiO2.p3}}+\Delta_{\mathrm{SiO2}} * \mathrm{M}_{\mathrm{Glass} .3}
\end{aligned}
$$

The final viscosity of the HLW Glass is given in Equation 49.

Equation 49

$\ln \left(\eta_{\text {Glass } . p 5}\right)=\sum_{i=1}^{19} c_{i} g_{i, p 5}+c_{20} g_{\text {Na2O }} g_{B 203 . p 5}+c_{21} g_{N a 2 O} g_{A l 203 . p 5}+c_{22} g_{B 2 O 3, p 5}^{2}+c_{23} g_{C a O . p 5} g_{A 12 O 3 . p 5}+c_{24} g_{\text {Li2O,p5 }}^{2}$

GlassProp $_{7}=7$

GO TO Product Consistency Test 